Submit FOR PUBLiCATion IN ApJS

Preprint typeset using LATEX style AASTeX6 v. 1.0

\title{
MPI-AMRVAC 2.0 FOR SOLAR AND ASTROPHYSICAL APPLICATIONS
}

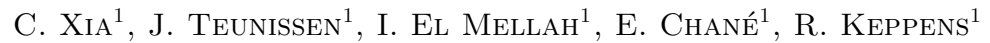

${ }^{1}$ Centre for mathematical Plasma Astrophysics, Department of Mathematics, KU Leuven, Celestijnenlaan 200B, 3001 Leuven, Belgium

\begin{abstract}
We report on the development of MPI-AMRVAC version 2.0, which is an open-source framework for parallel, grid-adaptive simulations of hydrodynamic and magnetohydrodynamic (MHD) astrophysical applications. The framework now supports radial grid stretching in combination with adaptive mesh refinement (AMR). The advantages of this combined approach are demonstrated with one-dimensional, two-dimensional and three-dimensional examples of spherically symmetric Bondi accretion, steady planar Bondi-Hoyle-Lyttleton flows, and wind accretion in Supergiant X-ray binaries. Another improvement is support for the generic splitting of any background magnetic field. We present several tests relevant for solar physics applications to demonstrate the advantages of field splitting on accuracy and robustness in extremely low plasma $\beta$ environments: a static magnetic flux rope, a magnetic null-point, and magnetic reconnection in a current sheet with either uniform or anomalous resistivity. Our implementation for treating anisotropic thermal conduction in multi-dimensional MHD applications is also described, which generalizes the original slope limited symmetric scheme from $2 \mathrm{D}$ to 3D. We perform ring diffusion tests that demonstrate its accuracy and robustness, and show that it prevents the unphysical thermal flux present in traditional schemes. The improved parallel scaling of the code is demonstrated with 3D AMR simulations of solar coronal rain, which show satisfactory strong scaling up to 2000 cores. Other framework improvements are also reported: the modernization and reorganization into a library, the handling of automatic regression tests, the use of inline/online Doxygen documentation, and a new future-proof data format for input/output.

Keywords: hydrodynamics — magnetohydrodynamics (MHD) — methods: numerical
\end{abstract}

\section{INTRODUCTION}

The solar and astrophysical research community can benefit from a whole array of publicly available software frameworks that are suited to model instrinsically multi-scale problems on modern computing infrastructure. A recurring ingredient in coping with the multi-scale nature is their capability of using adaptive mesh refinement (AMR). This is essential, e.g. in coping with Sun-to-Earth modeling of solar coronal mass ejections (Manchester et al. 2004), in many applications which extend magnetohydrodynamics (MHD) into special relativistic regimes (Mignone et al. 2012; Keppens et al. 2012), in applications recreating conditions in laser-plasma interactions (Tzeferacos et al. 2015), in simulations focusing on the formation and early evolution of disk galaxies in magnetized interstellar medium (Wang \& Abel 2009), or in fact in many problems where self-gravitational collapse is at play and AMR-MHD is combined with Poisson solvers (Ziegler 2005). AMR-MHD is currently available in a large - and still growing - variety of codes (e.g. BATS-R-US, Pluto, MPI-AMRVAC, FLASH, Enzo, Nirvana, Ramses, Athena, ASTRO-BEAR, ...), and it drove the development of novel MHD algorithms (e.g., Fromang et al. 2006; Rossmanith 2004; Stone et al. 2008; Cunningham et al. 2009). For example, recent Sun-to-Earth model efforts combine the use of a near-Sun overset Yin-Yang grid, with a cartesian AMR grid to handle the further heliosphere dynamics (Feng et al. 2011), where the AMR part is based on the PARAMESH (MacNeice et al. 2000) implementation.

In this paper, we report an upgrade to the open source MPI-AMRVAC software (Porth et al. 2014c), which was initially developed as a tool to study hyperbolic partial differential equation (PDE) systems such as the Euler system or ideal MHD with patch-based or hybrid-block-based AMR meshes (Keppens et al. 2003; van der Holst \& Keppens 2007). Using the loop annotation syntax (LASY) (Tóth 1997), the software is written in Fortran 90 plus Perl annotations so that the same code can be translated by a Perl preprocessor into any dimensional pure Fortran code before compilation. With the LASY implementation, typical expressions appearing in multidimensional simulation codes can be written in a much more concise form, less prone to mistakes. Adding geometric source terms, one-to-three dimensional AMR meshes 
can be in Cartesian, polar, cylindrical, or spherical coordinate systems. As a result, MPI-AMRVAC matured to a multiphysics software framework with modules to handle special relativistic hydro to MHD problems (Meliani et al. 2007; van der Holst et al. 2008; Keppens et al. 2012) and with the ability to couple different PDE models in localized domain regions across block-based AMR meshes (Keppens \& Porth 2014). To ensure efficient scaling on realistic applications, the AMR strategy evolved to a pure quadtree-octree block-based AMR hierarchy (Keppens et al. 2012; Porth et al. 2014c). In Porth et al. (2014c), we reported on conservative fourth-order finite difference schemes, and new modules to allow simulating hydrodynamics with dust coupling (Hendrix \& Keppens 2014) or Hall MHD (Leroy \& Keppens 2017). Selected applications of MPI-AMRVAC focus on shock-dominated special relativistic hydro problems in the context of gamma-ray burst blast waves and their afterglow (Meliani \& Keppens 2010; Vlasis et al. 2011), and up to full three-dimensional (3D) modeling of the precessing jets in the X-ray binary SS433 (Monceau-Baroux et al. 2014). Relativistic MHD applications studied propagation aspects of magnetized relativistic jets (Keppens et al. 2008), to detailed modeling of the Crab nebula (Porth et al. 2014b,a). Newtonian hydro applications addressed accretion processes onto compact objects (El Mellah \& Casse 2015), the wind collision and dust redistribution in the Wolf-Rayet 98a rotating pinwheel nebula (Hendrix et al. 2016), and MHD is employed in many solar challenges, such as modeling the in-situ formation of solar prominences (Xia et al. 2012, 2014; Xia \& Keppens 2016), solar coronal rain dynamics (Fang et al. 2013, 2015; Xia et al. 2017), or the initiation of coronal mass ejections (Zhao et al. 2017; Mei et al. 2017).

Recently, general relativistic fluid or plasma dynamics in arbitrary spacetimes can be handled by the Black Hole Accretion Code (BHAC), an MPI-AMRVAC spin-off that shares its algorithmic versatility and efficient block-based AMR ability (Porth et al. 2017). MPI-AMRVAC development focused on more Newtonian physics, with solar physics oriented functionality to extrapolate vector magnetic field distributions into coronal volumes using magnetofrictional methods (Guo et al. 2016c,b). This augments the earlier potential to linear-force-free field extrapolation in local Cartesian boxes or global spherical coordinates (Porth et al. 2014c), and paves the way to future data-driven solar applications. Driven by these ambitions as well as by several recent applications, many new functionalities have been developed. Since several of them can be of general interest to the astrophysical community and have not been documented elsewhere, we present them here in more detail, with an emphasis on demonstrating their advantages in a series of tests. We start with hydro to MHD applications where we employ radially stretched meshes in curvilinear coordinates in Section 2. This is shown to be crucial in accretion flow (or outflow) setups, where we wish to concentrate resolution around the accreting object near the inner radial boundary and alleviate the deformation of the cells at large radial distances. For MHD problems, the technique of magnetic field splitting, invented to handle low- $\beta$ shock-dominated plasma, was originally limited to potential (current-free) background fields (Tanaka 1994). We report an extension of this technique to allow any magnetic field to be the time-independent split-off part in Section 3. To accurately solve anisotropic thermal conduction in magnetized plasma, we present an extension to 3D of a slope-limited symmetric scheme in Section 4, which is shown to handle sharp temperature gradients very well. We report on scaling tests where these methods are adopted in full 3D coronal rain applications in Section 5. Other useful, more generic code framework improvements are reported in Section 6, which collectively define what we introduce as MPI-AMRVAC 2.0.

\section{RADIALLY STRETCHED MESHES}

Many physical problems rely on the use of spherical, polar or cylindrical meshes, and at the same time intend to monitor the evolution of gas or plasma flows over several spatial orders of magnitude. In astrophysical configurations, the interplay between gravitation and in- or outflows is a recurring ingredient that would benefit from combining e.g. a spherical mesh with a gradual radial stretching of the cells, in combination with block-adaptivity. Here, we report on one-dimensional (1D) to fully $3 \mathrm{D}$ tests or applications where this is exploited.

\subsection{D Bondi transonic accretion}

The simplest hydrodynamical representation of the accretion of matter by a point mass has been analysed early on by Bondi (1952). It is an isotropic problem where only radial dependencies and components are considered. It describes how a non-zero temperature flow moving at a subsonic speed at infinity towards a central object of mass $M_{*}$ can establish a steady, smooth and transonic inflow solution. This unique continuous, transonic inflow solution $v_{r}(r)$ does not display any shock. Provided the evolution is adiabatic, it means that the flow is isentropic: the pressure $p(r)$ can be deduced from the mass density $\rho(r)$, using the fixed adiabatic index $\gamma$ of the flow $\left(p \rho^{-\gamma}\right.$ is independent of radius $r$ ). It is convenient to adopt the following normalization quantities:

1. the mass density at infinity, $\rho_{\infty}$, 
2. the sound speed at infinity, $c_{\infty}$,

3. the radius $R_{0}=2 G M_{*} / c_{\infty}^{2}$, where $M_{*}$ is the mass of the accretor and $G$ the gravitational constant.

Using these scalings, the mass per unit time crossing a sphere of a given radius (the mass accretion rate, $\dot{M}$ ) and the pressure are normalized with $\rho_{\infty} c_{\infty} R_{0}^{2}$ and $\rho_{\infty} c_{\infty}^{2}$, respectively. From now on, we work with normalized dimensionless variables. The analytic expressions below assume the specific case of a zero speed at infinity, but the conclusions below remain qualitatively the same in the general case.

The fundamental equations of this problem are the mass continuity equation

$$
\frac{\partial \rho}{\partial t}+\frac{1}{r^{2}} \frac{\partial\left(r^{2} \rho v_{r}\right)}{\partial r}=0,
$$

linear momentum conservation with gravity as a source term

$$
\frac{\partial\left(\rho v_{r}\right)}{\partial t}+\frac{1}{r^{2}} \frac{\partial\left(r^{2} \rho v_{r}^{2}\right)}{\partial r}+\frac{\partial p}{\partial r}=-\frac{\rho}{2 r^{2}},
$$

and the isentropic relation between (scaled) pressure and density as $p \rho^{-\gamma}=p_{\infty} /\left(\rho_{\infty} c_{\infty}^{2}\right)=1 / \gamma$. The steady-state solution admits a radius at which it becomes supersonic, called the sonic radius $r_{s}$, whose expression can be analytically derived to be $r_{s}=(5-3 \gamma) / 8$. The isothermal case (i.e. $\gamma=1$ ) has its associated sonic radius at $r_{s, 0}=1 / 4$ (this is identical for the corresponding unique outflowing Parker isothermal wind solution). The normalized mass accretion rate can also be determined from the adiabatic index:

$$
\dot{M}=\frac{\pi}{4}\left(\frac{2}{5-3 \gamma}\right)^{\frac{5-3 \gamma}{2(\gamma-1)}} .
$$

For derivations of these results above, in the more general case of a non zero subsonic speed at infinity, the reader might refer to Section 4.2 of El Mellah (2016). Below $r=1$ (i.e. below the position of the scaling radius $R_{0}$ ) the properties of the flow significantly depart from their values at infinity. For adiabatic indexes close to the maximum value of $5 / 3$ that it can take, the sonic radius $r_{s}$ becomes very small. We want to have the sonic radius within the simulation space, to have a trivial inner boundary treatment where inflow is supersonic. It then becomes advantagous to resort to a stretched mesh.

We work on domains extending from a radius of 0.01 (inner boundary) to 10 (outer boundary). We rely on a second order two-step HLLC method (Toro et al. 1994), both for the predictor and the full time step, with the Koren slope limiter (Vreugdenhil \& Koren 1993) used in the limited reconstruction of the primitive variables $\rho$ and $v_{r}$, to obtain cell-face values. To design our initial conditions and fix the outer $r=10$ inflow boundary conditions, we reformulate the fundamental equations to obtain a transcendental equation which indirectly gives the mass density as a function of radius $r$

$$
\frac{\rho^{\gamma-1}-1}{\gamma-1}-\frac{1}{2 r}+\frac{\dot{M}^{2}}{32 \pi^{2}} \frac{1}{\rho^{2} r^{4}}=0 .
$$

We numerically solve this relation with a Newton-Raphson method, which behaves correctly except very near the sonic radius where the algorithm may yield non monotonous results. In this restricted region, we compute the mass density profile by linearly interpolating between points further away from the sonic radius. The obtained mass density profile is used as initial condition and sets the fixed mass density in the ghost cells beyond $r=10$. For the linear momentum $\rho v_{r}$, we deduce its radial profile from Eq. (3) and similarly implement it both as initial condition and as forced outer boundary condition. For the inner boundary conditions, we copy the mass density in the ghost cells and then guarantee the continuity of the mass flux and Bernoulli invariant (as in El Mellah \& Casse 2015). We then use MPI-AMRVAC to time-advance the solution to retrieve the stationary analytical solution, on three different kinds of radial spherical meshes :

1. a non stretched grid with a total number of cells $N=16388$, without AMR,

2. a stretched grid with a much reduced total number of cells $(N=132)$, stretched such that the relative resolution at the inner boundary remains the same as for the previous grid, without AMR,

3. the same stretched grid as above, but with a total of 5 levels of mesh refinement, where blocks which contain the analytic sonic radius are refined. The refined grid is computed at the initial state and does not evolve afterwhile (static AMR). 

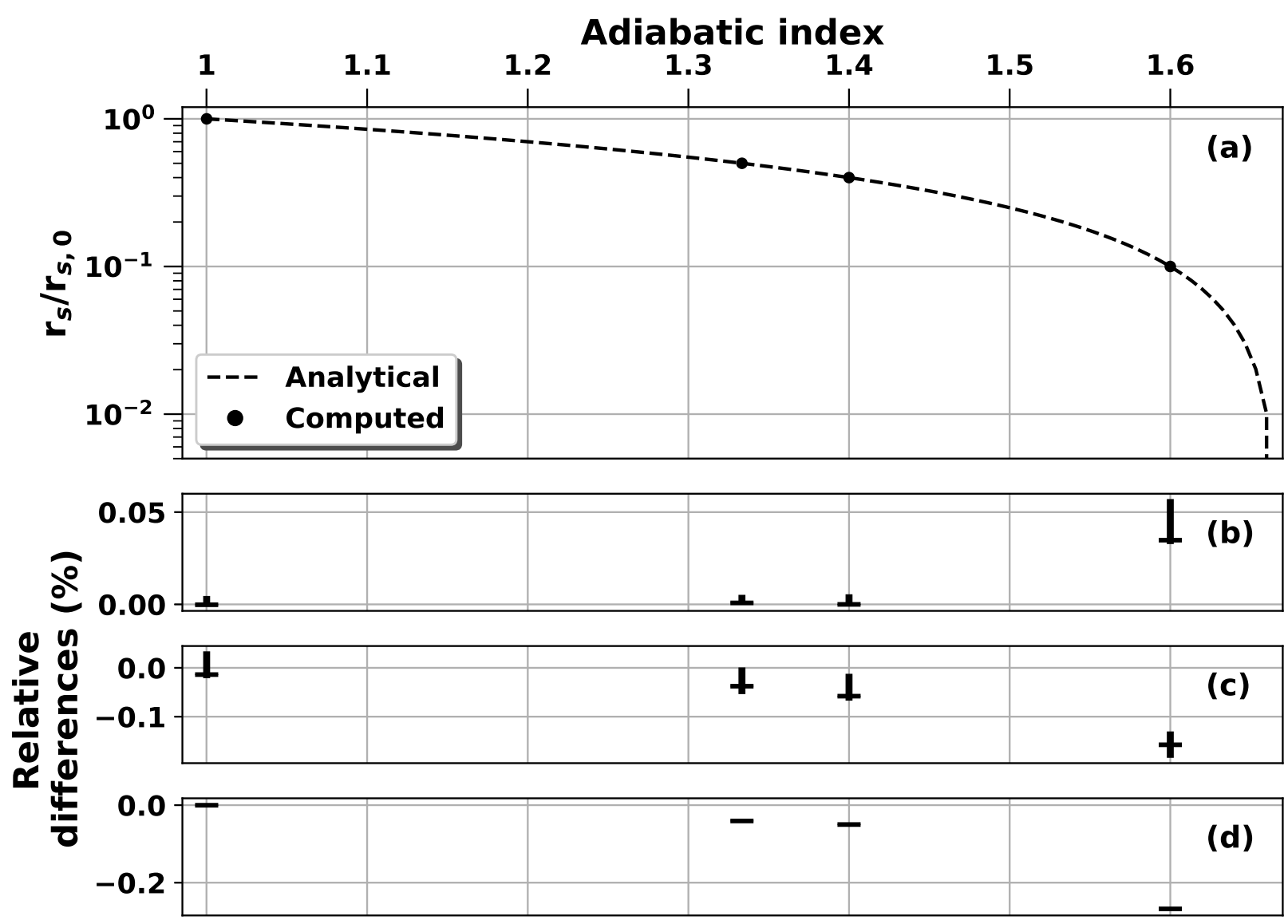

Figure 1. (a) Sonic radius $r_{s}$ relative to its value $r_{s, 0}$ at $\gamma=1$ as a function of the adiabatic index. The dashed line represents the analytic solution given by $r_{s}=(5-3 \gamma) / 8$, while the 4 dots are the sonic radii measured from the steady-state solution computed by MPI-AMRVAC. (b,c,d) Relative differences between the analytic and computed sonic radii for a non-stretched high resolution grid, a stretched grid and a stretched grid with AMR.

Within a few dynamical times, the profiles relax towards a numerical equilibrium close to the solution determined with the Newton-Raphson above. To quantify the relaxed steady states, we measure the position of the sonic radius obtained for each of the three meshes, and this for 4 different adiabatic indexes. The results are summarized in Figure 1. By eye, it is impossible to distinguish the numerically obtained sonic radii (dots) from the analytic solution (dashed curve) displayed in the upper panel, whatever the mesh. Therefore, we plotted the relative differences in the three lower panels for the non stretched grid (b), a stretched grid (c) and a stretched grid with AMR (d). The error bars are evaluated by considering one hundredth of the spatial resolution in the vicinity of the sonic radius. We can conclude from those results that analytic sanity checks such as the position of the sonic radius are retrieved within a few tenths of percent with MPI-AMRVAC.

Apart from this local agreement on the sonic point location, we can also quantify an error estimate for the entire relaxed mass density profiles obtained. For the case where $\gamma=1.4$, we compare runs with different AMR levels employing stretched meshes, to the relaxed mass density profile obtained with the non-stretched, high resolution mesh above. We computed the $L_{2}$-norm between data points on stretched, AMR meshes and their counterpart on the non-stretched mesh (linearly interpolated to the same radial positions). Figure 2 shows that the matching between the solutions obtained increases as the number of maximum AMR levels rises.

\subsection{Radial stretching principle}




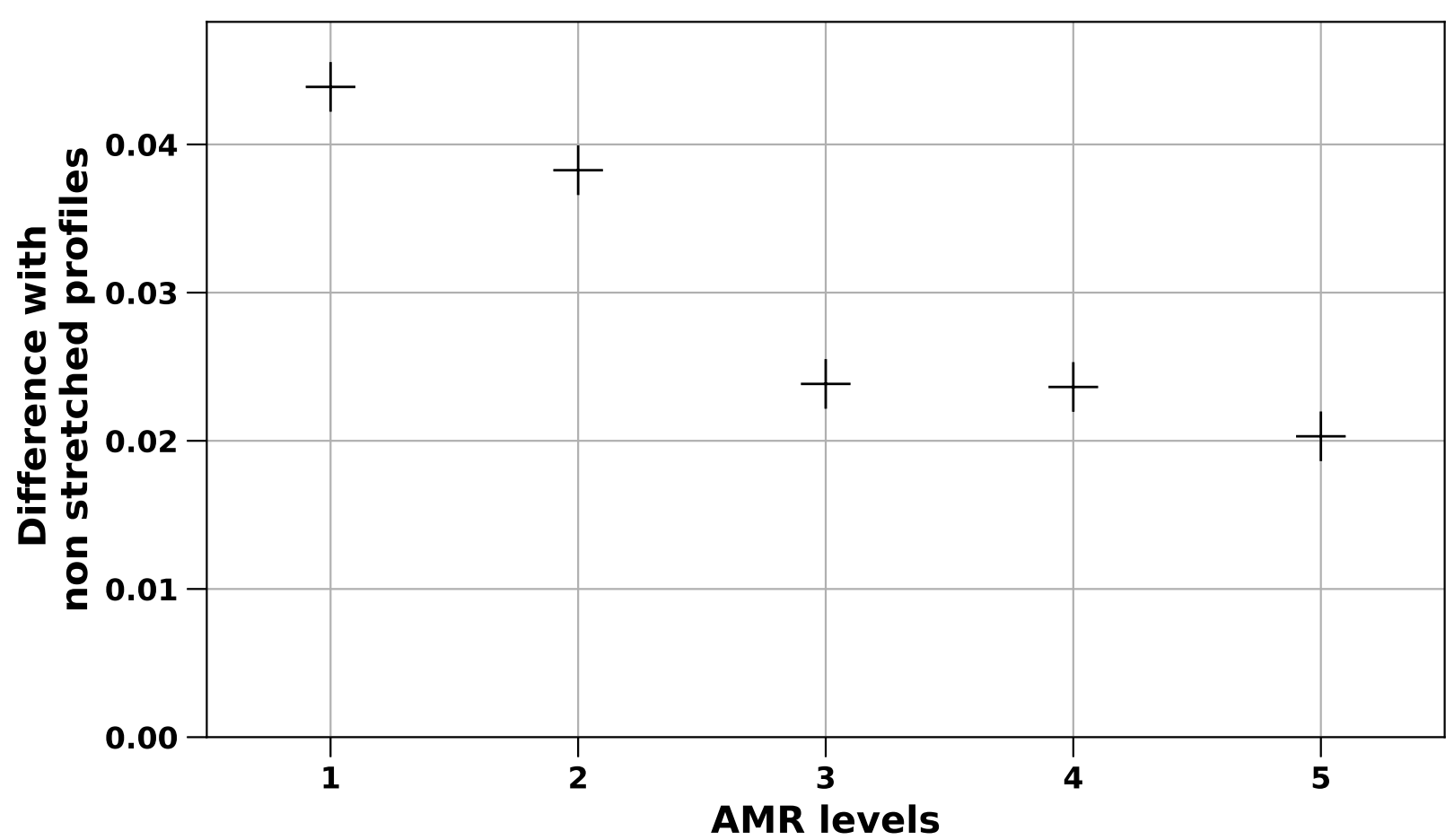

Figure 2. Quantification of the departure of the relaxed mass density profiles obtained with stretched meshes, for different AMR levels, with respect to the profile obtained with a non stretched mesh. Those results were obtained for $\gamma=1.4$.

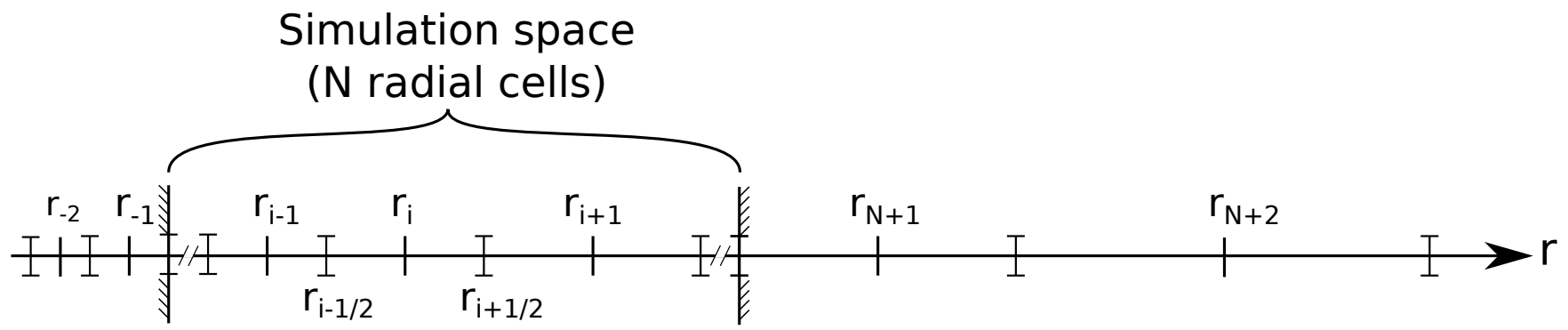

Figure 3. Uni-dimensional representation of the radial stretching with the cell centers marked with a vertical line and the cell edges with a back-to-back double bracket. The cell centers are always mid-way between two consecutive edges, but the reverse is not true. The inner and outer edges are marked by longer vertical lines with dashes. There are two ghost cells on each side $(n=2)$ in this example.

The radial stretching is introduced both to decrease the number of radial cells and, in two-dimensional (2D) and 3D, to alleviate the deformation of cells when the radius of the inner radial boundary of the grid is orders of magnitude smaller than the radius of the outer boundary. To keep a constant cell aspect ratio all over a $2 \mathrm{D}(r, \theta)$ grid, the radial step size $\Delta r_{i}$ must obey the following relation :

$$
\frac{\Delta r_{i}}{r_{i} \Delta \theta}=\zeta
$$

where $r_{i}$ is the local radius, $\Delta \theta$ the uniform angular step (the latitudinal one for a spherical $(r, \theta, \varphi)$ mesh where $\theta$ is the colatitude) and $\zeta$ is a fixed aspect ratio. Since the radial step is then proportional to the radius, we obtain a kind of self-similar grid from the innermost to the outermost regions. We set the radial position $r_{i}$ of the center of any cell indexed $i$ mid-way between the two surrounding interfaces, $r_{i-1}$ and $r_{i+1}$, see Figure 3 . The recursive relationship 
which builds up the sequence of radial positions for the cell centers is given by :

$$
r_{i+1}=r_{i}+\frac{\Delta r_{i}}{2}+\frac{\Delta r_{i+1}}{2}
$$

where $\Delta r_{i}$ is the radial step of cell $i$. Inserting equation (5) yields :

$$
r_{i+1}=q r_{i} \quad \text { where } \quad q=\frac{1+\zeta \Delta \theta / 2}{1-\zeta \Delta \theta / 2} .
$$

Hereafter, $q$ is called the scaling factor. We fix the radii of the inner and outer radial boundaries of the domain, $r_{i n}$ and $r_{\text {out }}$ respectively, along with the total number of cells in the radial direction $N$ (excluding the ghost cells). The scaling factor is then computed from equation (7) applied to the two boundaries: $q=\left(r_{\text {out }} / r_{\text {in }}\right)^{1 / N}$. We can derive the required aspect ratio for a radially stretched grid :

$$
\zeta=\frac{2(q-1)}{\Delta \theta(q+1)}
$$

For a stretching smooth enough and a usual number (typically 2) of ghost cells, the radial positions of the inner ghost cells remain positive.

As should be clear from our example applications, the stretched grid is created accordingly to the type of symmetry (cylindrical or spherical) and to the dimensionality (1D, 2D or 3D). The numerical schemes incorporate the variable radial step size where needed. The coupling between stretched grids and block-based AMR has been made possible by computing a scaling factor for each AMR level. Since children blocks at the next level span the same radial range as the mother block but with twice as many cells, the scaling factor $q_{l+1}$ of the children blocks at level $l+1$ can be obtained from the scaling factor $q_{l}$ of the mother block at level $l$ via $q_{l+1}=\sqrt{q_{l}}$.

The time gain can be estimated by writing the ratio of the number of radial cells in a non-stretched versus a stretched grid, with the same absolute radial step at the inner boundary. It approximately amounts to :

$$
\Lambda \sim \frac{r_{\text {out }} / r_{\text {in }}}{2 \log _{10}\left(r_{\text {out }} / r_{\text {in }}\right)}
$$

which quickly rises when $r_{\text {out }} / r_{\text {in }}$ exceeds a few tens. Moreover, in combination with AMR, the stretched grid enables to resolve off-centered features (see e.g. our 3D application in Section 2.4), without lowering the time step, if the latter is dominated by dynamics within the innermost regions. In the previous section on 1D Bondi accretion, the number of cells for an accurate solution could be reduced by two orders of magnitude.

\subsection{The 2.5D Bondi-Hoyle-Lyttleton problem}

A truly two-dimensional accretion-type problem is the Bondi-Hoyle-Lyttleton (BHL) model designed by Hoyle \& Lyttleton (1939) and Bondi \& Hoyle (1944) (for a more detailed review of the BHL model and its refinements, see Edgar 2004; El Mellah 2016). The major change with respect to the 1D Bondi flow is that there is now a supersonic bulk planar motion of the flow with respect to the accretor, at speed $v_{\infty}>c_{\infty}$, which has two serious consequences:

1. the geometry of the problem is no longer isotropic but is still axisymmetric (around the axis through the accretor and with direction set by the speed of the inflow at infinity),

2. since the flow is supersonic at infinity, there can be a bow shock induced by the interaction with the accretor. The isentropic assumption we used to bypass the energy equation in section 2.1 no longer holds.

Analytic considerations point to a formation of the shock at a spatial scale of the order of the accretion radius $R_{a c c}=2 G M_{*} / v_{\infty}^{2}$. This radius is close to the size of the accretor, when we study stars accreting the wind of their companion in binary systems (Chen et al. 2017). However, for compact accretors which have an associated Schwarzschild radius $R_{s c h}=2 G M_{*} / c^{2}$, the contrast between accretor size and the scale at which the flow is deflected by the gravitational influence of the accretor becomes important, since

$$
\frac{R_{a c c}}{R_{s c h}}=\left(\frac{c}{v_{\infty}}\right)^{2},
$$

where $c$ is the speed of light. For realistic speeds $v_{\infty}$ of stellar winds in wind-dominated X-ray binaries for instance, it means that the accretor is 4 to 5 orders of magnitude smaller than the shock forming around it. To bridge the gap between those scales, a stretched grid is mandatory. 

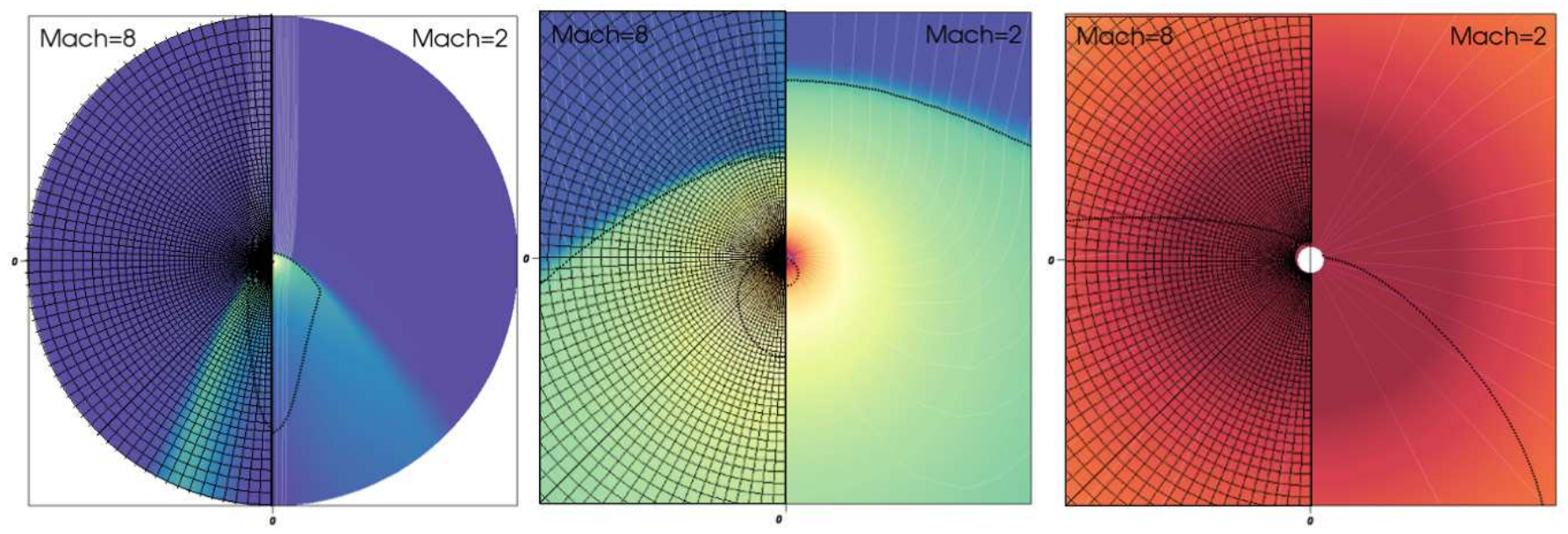

Figure 4. Logarithmic colour maps of the density for Mach numbers at infinity of 8 (left panels) and 2 (right panels). Some streamlines have been represented (solid white) and the two dotted black contours stand for the Mach-1 surface. Zoom in on the central area by a factor of 20 (center) and 400 (right).

This has been done with MPI-AMRVAC in El Mellah \& Casse (2015), where we solved the hydro equations on a 2D $(r, \theta)$ grid combined with axisymmetry about the ignored $\varphi$ direction. The set of equations to solve now include the energy equation, also with work done by gravity appearing as a source term, where the energy variable $E=$ $p /(\gamma-1)+\rho\left(v_{r}^{2}+v_{\theta}^{2}\right) / 2$ is used to deduce the pressure. In the simulations, we work with an adiabatic index representative of a monoatomic gas, $\gamma=5 / 3$. The equations solved write as:

$$
\begin{gathered}
\frac{\partial \rho}{\partial t}+\frac{1}{r^{2}} \frac{\partial\left(r^{2} \rho v_{r}\right)}{\partial r}+\frac{1}{r \sin \theta} \frac{\partial\left(\rho v_{\theta} \sin \theta\right)}{\partial \theta}=0 \\
\frac{\partial\left(\rho v_{r}\right)}{\partial t}+\frac{1}{r^{2}} \frac{\partial\left(r^{2} \rho v_{r}^{2}\right)}{\partial r}+\frac{1}{r \sin \theta} \frac{\partial\left(\rho v_{r} v_{\theta} \sin \theta\right)}{\partial \theta}+\frac{\partial p}{\partial r}=\frac{\rho v_{\theta}^{2}}{r}-\frac{\rho}{2 r^{2}}, \\
\frac{\partial\left(\rho v_{\theta}\right)}{\partial t}+\frac{1}{r^{2}} \frac{\partial\left(r^{2} \rho v_{r} v_{\theta}\right)}{\partial r}+\frac{1}{r \sin \theta} \frac{\partial\left(\rho v_{\theta}^{2} \sin \theta\right)}{\partial \theta}+\frac{1}{r} \frac{\partial p}{\partial \theta}=-\frac{\rho v_{r} v_{\theta}}{r}, \\
\frac{\partial E}{\partial t}+\frac{1}{r^{2}} \frac{\partial\left(r^{2}(E+p) v_{r}\right)}{\partial r}+\frac{1}{r \sin \theta} \frac{\partial\left[(E+p) v_{\theta} \sin \theta\right]}{\partial \theta}=-\frac{\rho v_{r}}{2 r^{2}} .
\end{gathered}
$$

The terms on the RHS are handled in geometric and gravitational source terms.

Fortunately, not all quantities require an inner $r_{i n}$ boundary which matches the physical size of the accretor. For instance, Ruffert (1994) quantified the impact of the size of the inner boundary $r_{i n}$ on the measured mass accretion rates at the inner boundary, $\dot{M}_{i n}$ (see Figure 5.3 in El Mellah 2016). The result is that $\dot{M}_{i n}$ matches the analytic value provided one reaches the sonic radius. But as explained in the previous $1 \mathrm{D}$ Bondi test, and as can be seen in Figure 1, the sonic radius drops to zero for $\gamma=5 / 3$. Ruffert (1994) showed that to retrieve the analytic value of the mass accretion rate within a few percent, $r_{i n}$ must be below a hundredth of the accretion radius. Therefore, in the simulations where $\gamma=5 / 3$, we need to span at least 3 orders of magnitude: while the outer boundary lies at $8 R_{a c c}$, the inner boundary has a radius of a thousand of the accretion radius. With a resolution of $176 \times 64$ cells in the $(r, \theta)$ plane (without the ghost cells), it gives an aspect ratio of 1.04 (and a scaling factor of 1.05).

The steady solution has the supersonic planar outflow upstream, suddenly transitioning to a subsonic inflow at the bow shock. Then, part of this now subsonic inflow is still accreted in a continuous Bondi-type transonic fashion in the wake of the accretor. Solutions for different Mach numbers $\mathcal{M}_{\infty}=v_{\infty} / c_{\infty}$ of the supersonic planar inflow are compared in Figure 4, showing density variations in a zoomed in fashion. The upstream conditions at $8 R_{a c c}$ are set by the ballistic solutions (Bisnovatyi-Kogan et al. 1979) and the downstream boundary condition is continuous (with a supersonic outflow). At the inner boundary, we enforce the same boundary conditions as described in section 2.1, with a continuous $v_{\theta}$. MPI-AMRVAC provides a steady solution on a stretched (non AMR) grid which matches the orders of magnitude mentioned above: the position of the detached bow shock, the jump conditions at the shock, as well as the inner mass accretion rate (El Mellah \& Casse 2015). In particular, we retrieve a topological property derived by Foglizzo \& Ruffert (1996) concerning the sonic surface, deeply embedded in the flow and anchored into the inner boundary, whatever its size (see the solid black contour denoting its location in the zoomed views in Figure 4). 


\subsection{D Clumpy wind accretion in Supergiant X-ray Binaries}

A third hydrodynamic application of radially stretched AMR grids generalizes the 2D (spherical axisymmetric) setup above to a full 3D case. In a forthcoming paper (El Mellah et al., in prep.), we use a full 3D spherical mesh to study wind-accretion in Supergiant X-ray Binaries, where a compact object orbits an evolved mass-losing star. In these systems, the same challenge of scales identified by equation (10) is at play, and the BHL shock-dominated, transonic flow configuration is applicable. In realistic conditions, the wind of the evolved donor star is radiatively driven and clumpy, making the problem fully time-dependent. We model the accretion process of these clumps onto the compact object, where the inhomogeneities (clumps) in the stellar wind enter a spherically stretched mesh centered on the accreting compact object, and the clump impact on the time-variability of the mass accretion rate is studied. The physics details will be provided in that paper, here we emphasize how the $3 \mathrm{D}$ stretched AMR mesh is tailored to the problem at hand. The initial and boundary conditions are actually derived from the $2.5 \mathrm{D}$ solutions described above, while a seperate 2D simulation of the clump formation and propagation in the companion stellar wind serves to inject time-dependent clump features in the supersonic inflow.

The use of a 3D spherical mesh combined with explicit time stepping brings in additional challenges since the time step $\Delta t$ must respect the Courant-Friedrich-Lewy (CFL) condition. Since the azimuthal line element is $r \sin \theta \Delta \varphi$ with colatitude $\theta$, it means that the time step obeys:

$$
\Delta t(\theta) \propto \sin (\theta) .
$$

The axisymmetry adopted in the previous section avoided this complication. As a consequence, the additional cost in terms of number of iterations to run a $3 \mathrm{D}$ version of the setup presented in the previous section is approximately:

$$
\frac{\Delta t(\pi / 2)}{\Delta t\left(\theta_{\min }\right)} \sim \frac{1}{\theta_{\min }}
$$

where $\theta_{\min } \ll \pi / 2$ is the colatitude of the first cell center just off the polar axis. For a resolution on the base AMR level of 64 cells spanning $\theta=0$ to $2 \pi$, it means a time step 40 times smaller compared to the two-dimensional counterpart. Together with the additional cells in the azimuthal direction, this makes a 3D setup much more computationally demanding.

We compromise on this aspect by using an AMR mesh which deliberately exploits a lower resolution at the poles, while still resolving all relevant wind structures. Since the clumps embedded in the supersonic inflow are small scale structures, we need to enable AMR (up to 4 levels in the example of Figure 5) in the upstream hemisphere to resolve them, in particular at the outer edge of the mesh where the radially stretched cells have the largest absolute size on the first AMR level. At the same time, we are only interested in the fraction of the flow susceptible to be accreted by the compact object, so we can inhibit AMR refinement in the downstream hemisphere, except in the immediate vicinity of the accretor (below the stagnation point in the wake). We also prevent excessive refinement in the vicinity of the accretor (no refinement beyond the third level in Figure 5) since the stretching already provides more refined cells. Finally, in the upstream hemisphere, we favor AMR refinement in the accretion cylinder, around the axis determined by the direction of the inflow velocity. Note that this axis was the symmetry polar axis in the previous $2.5 \mathrm{D}$ setup in section 2.3, but this is different in the present 3D setup, where the polar axis is above and below the central accretor. In the application to study supergiant X-ray binary accretion, together with geometrically enforced AMR, we use dynamic AMR with a refinement criterion on the mass density to follow the flow as it is accreted onto the compact object.

\subsection{Trans-Alfvénic solar wind}

To simulate the propagation of the solar wind between the Sun and the orbit of the Earth (at $215 \mathrm{R}_{\odot}$ ), the use of a spherical stretched grid can also be very important. First, it considerably reduces the amount of cells needed in the simulations (by a factor of 46). Second, it allows to maintain the same aspect ratio for all the cells in the simulation. We present here the results of 2.5D MHD simulations of the meridional plane of the solar wind, i.e., axisymmetric simulations on a 2D mesh where the 3 vector components are considered for the plasma velocity and for the magnetic field. This simulation setup also allows us to test the magnetic field splitting (MFS) method discussed in Section 3. Instead of solving the induction equation for the total magnetic field $(\mathbf{B})$, one can solve it for the difference with the internal dipole field of the Sun $\left(\mathbf{B}_{\mathbf{1}}=\mathbf{B}-\mathbf{B}_{\mathbf{0}}\right)$, where $\mathbf{B}_{\mathbf{0}}$ represent the dipole field. We will here compare the results of these two setups. In our simulations, the solar wind is generated through the inner boundary (at $r=1 \mathrm{R}_{\odot}$ ), in a fashion similar to Jacobs et al. (2005); Chané et al. $(2005,2008)$ : the temperature is fixed to $1.5 \times 10^{6} \mathrm{~K}$, and the number density to $7.1 \times 10^{6} \mathrm{~cm}^{-3}$, where the azimuthal velocity is set to $\left(2.09-0.264 \cos ^{2} \theta-0.334 \cos ^{4} \theta\right) \sin \theta \mathrm{km}$ 


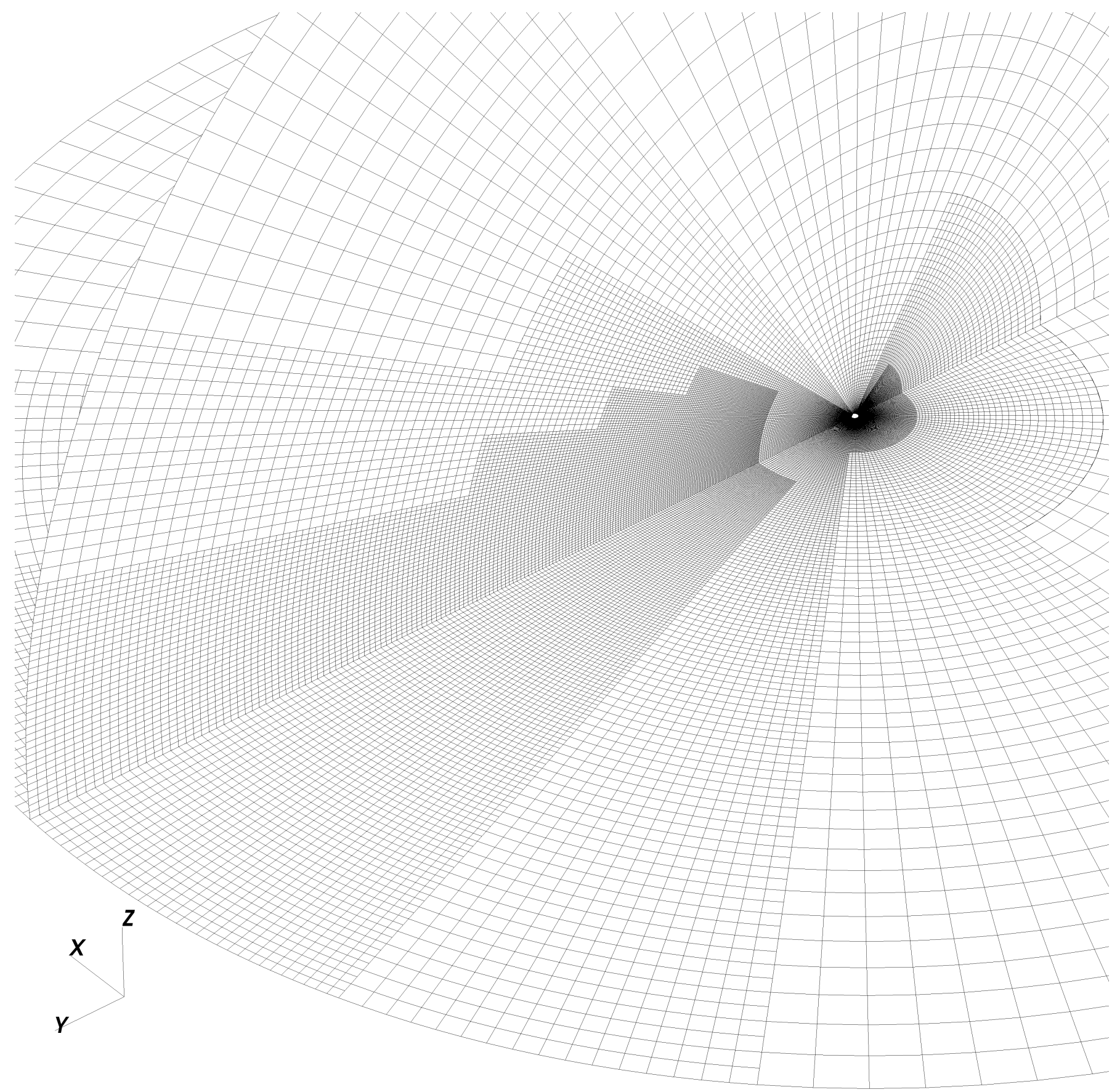

Figure 5. Two slices of the upper hemisphere of a spherical mesh with 4 levels of refinement, as used in a study of time-dependent wind-accretion onto a compact object. User-defined restrictions on AMR prevent the grid to be refined in some areas (e.g. in the downstream hemisphere, where the $y$-coordinate is negative) far from the inner boundary and in the vicinity of the polar $z$-axis). We further restrict the maximum level of refinement to 3 in the vicinity of the inner boundary and allow for maximum refinement in the outer regions of the upstream hemisphere close to the $y$-axis. This corresponds to the supersonic inflow area where clumps enter the grid.

$\mathrm{s}^{-1}$ to enforce the solar differential rotation of the Sun ( $\theta$ is the co-latitude), and where the radial component of the magnetic field is fixed to the internal dipole value (with $B_{r}=1.1 \mathrm{G}$ at the equator). In addition, close to the equator (at latitudes lower than $22.6^{\circ}$ ), in the streamer region, a dead-zone is enforced at the boundary by fixing the radial and the latitudinal velocities to zero. The equations solved are the ideal MHD equations plus two source terms: one for the heating of the corona and one for the gravity of the Sun. The heating of the corona is treated as an empirical 

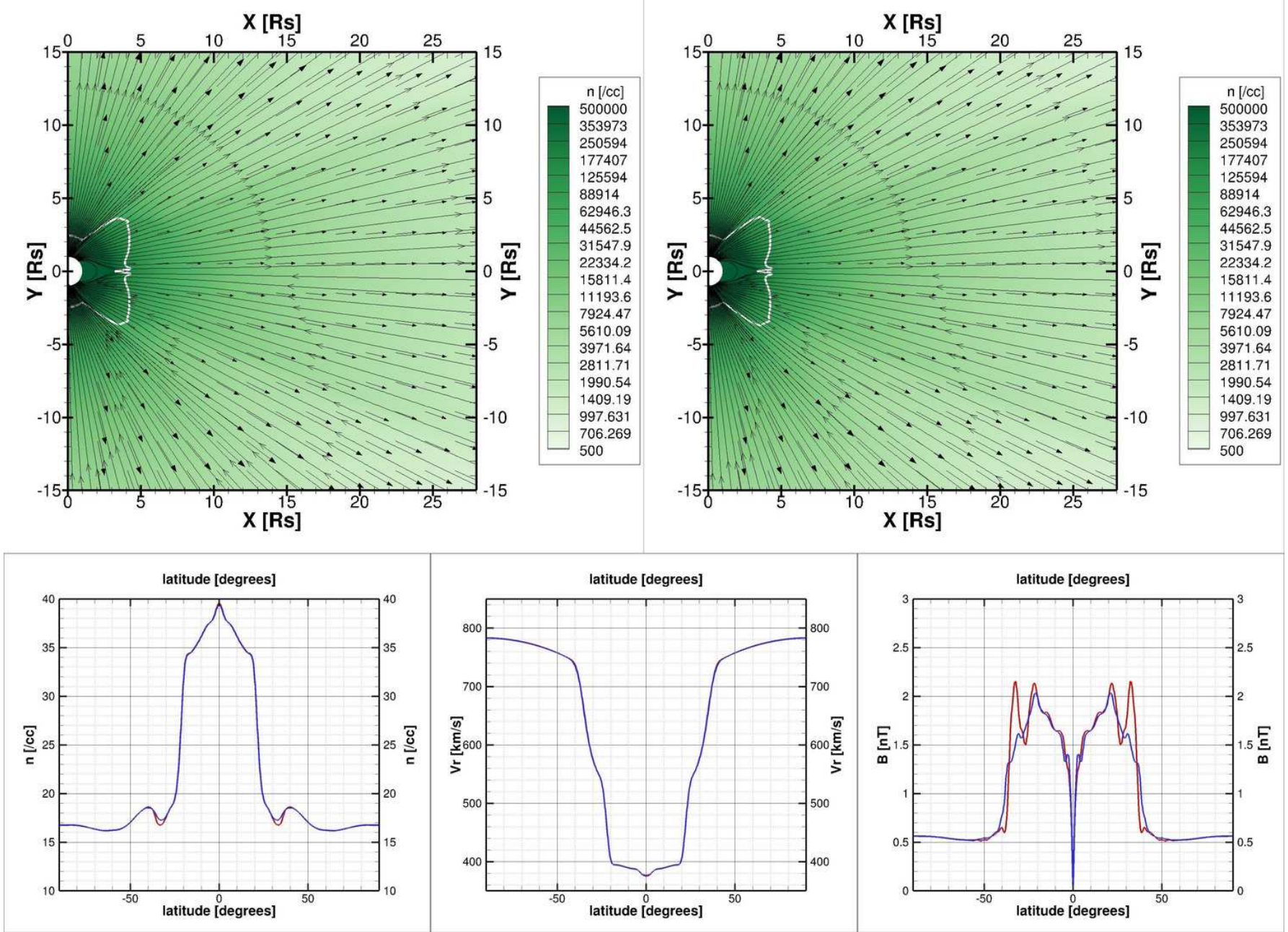

Figure 6. Axisymmetric 2.5D MHD simulations of the solar wind. The top panels show the color-coded density, the velocity vectors, the magnetic field lines (in black), as well as the Alfvén surface (white line) for two simulations: one where the magnetic field splitting method is used (right panel) and one where it is not (left panel). The lower panels show the solar wind characteristics at 1 AU: the plasma number density (left panel), radial velocity (middle panel), and the magnetic field magnitude (right panel) for both simulation (with MFS in red and without MFS in blue).

source term in the energy equation. We follow the procedure described in Groth et al. (2000) with a heating term dependent on the latitude (with a stronger heating at high latitudes) and on the radial distance (the heating decreases exponentially). The inclusion of this source term result in a slow solar wind close to the equatorial plane and a fast solar wind at higher latitudes (see Figure 6)

The simulations are performed on a spherical stretch mesh between $1 R_{\odot}$ and $216 R_{\odot}$ with 380 cells in the radial direction and 224 cells in the latitudinal direction. For these simulations, we do not take advantage of the AMR capabilities of the code and one single mesh level is used (no refinement). The smallest cells (close to the sun) are $0.014 \mathrm{R}_{\odot}$ large, while the largest cells (at $1 \mathrm{AU}$ ) are $3 \mathrm{R}_{\odot}$ large. The equations are solved with a finite-volume scheme, using the two-step HLL solver (Harten et al. 1983) with Koren slope limiters. Powell's method (Powell et al. 1999) is used for the divergence cleaning of the magnetic field. The adiabatic index $\gamma$ is set to $5 / 3$ in these simulations.

The results of the simulations are shown in Figure 6. The top panels display the plasma density, the velocity vectors, the magnetic field lines, and the Alfvén surface. One can see that both panels are very similar with: 1) a fast solar wind at high latitudes and a slow denser wind close to the equatorial plane, 2) a dense helmet streamer at low latitudes close to the Sun where the plasma velocity is almost zero, 3) an Alfvén surface located between 2 and 7 R $\odot$ in both cases. To highlight the differences between the two simulations, one dimensional cuts at $1 \mathrm{AU}$ are provided in the lower panels of Figure 6 for both simulations. The density profiles are very similar, with only small differences at the transition between the slow and the fast solar winds. For both simulations, the solar wind is, as expected, denser 
close to the equatorial plane. The radial velocity profiles (shown in the middle panel) are nearly identical in both simulations, with a fast solar wind (a bit less than $800 \mathrm{~km} \mathrm{~s}^{-1}$ ) at high latitudes, and a slow solar wind (a bit less than $400 \mathrm{~km} \mathrm{~s}^{-1}$ ) close to the equator. These results are very similar to in situ measurements at $1 \mathrm{AU}$ (see, for instance, Phillips et al. 1995). The differences are more pronounced for the magnetic field magnitude (right panel), where the magnetic field can be up to $30 \%$ stronger in a small region located at the transition region between the fast and slow solar winds when the MFS method is used. In general, the differences between the two simulations are negligible and confined to small specific areas of the simulations.

\section{MAGNETIC FIELD SPLITTING}

When numerically solving for dynamics in solar or planetary atmospheres, the strong magnetic field and its gradients often cause serious difficulties if the total magnetic field is solved for as a dependent variable. This is especially true when both high- and low- $\beta$ regions need to be computed accurately in shock-dominated interactions. An elegant solution to this problem (Tanaka 1994) is to split the magnetic field $\mathbf{B}=\mathbf{B}_{\mathbf{0}}+\mathbf{B}_{\mathbf{1}}$, into a dominating background field $\mathbf{B}_{\mathbf{0}}$ and a deviation $\mathbf{B}_{\mathbf{1}}$, and solve a set of modified equations directly quantifying the evolution of $\mathbf{B}_{\mathbf{1}}$. Splitting off this background magnetic field component and only evolving the perturbed magnetic field is more accurate and robust, in the sense that it diminishes the artificial triggering of negative gas pressure in extremely low- $\beta$ plasma. This background field $\mathbf{B}_{\mathbf{0}}$ was originally limited to be a time-independent potential field (Tanaka 1994) (as used in the previous section for the solar wind simulation on a stretched mesh), and in this form has more recently led to modify the formulations of HLLC (Guo 2015) or HLLD (Guo et al. 2016a) MHD solvers, which rely on a 4 to 6-state simplification of the local Riemann fans, respectively. The splitting can also be used for handling background timedependent, potential fields (Tóth et al. 2008), even allowing for extensions to Hall-MHD physics where challenges due to the dispersive whistler wave dynamics enter. The governing equations for a more general splitting, as revisited below, were also presented in Gombosi et al. (2002), giving extensions to semi-relativistic MHD where the Alfvén speed can be relativistic. The more general splitting form also appears in Feng et al. (2011), although they further apply it exclusively with time-independent, potential fields.

\subsection{Governing equations, up to resistive MHD}

Here, we demonstrate this MFS method in applications that allow any kind of time-independent magnetic field as the background component, no longer restricted to potential (current-free) settings. At the same time, we wish to also allow for resistive MHD applications that are aided by this more general MFS technique. The derivation of the governing equations employing an arbitrary split in a time-independent $\mathbf{B}_{\mathbf{0}}$ are repeated below, to extend them further on to resistive MHD. The ideal MHD equations in conservative form are given by

$$
\begin{aligned}
\frac{\partial \rho}{\partial t}+\nabla \cdot(\rho \mathbf{v}) & =0 \\
\frac{\partial(\rho \mathbf{v})}{\partial t}+\nabla \cdot\left(\rho \mathbf{v} \mathbf{v}+\left(p+\frac{\mathbf{B} \cdot \mathbf{B}}{2 \mu_{0}}\right) \mathbf{I}-\frac{\mathbf{B B}}{\mu_{0}}\right) & =0 \\
\frac{\partial E}{\partial t}+\nabla \cdot\left(E \mathbf{v}+\left(p+\frac{\mathbf{B} \cdot \mathbf{B}}{2 \mu_{0}}\right) \mathbf{v}-\frac{\mathbf{B}}{\mu_{0}}(\mathbf{B} \cdot \mathbf{v})\right) & =0 \\
\frac{\partial \mathbf{B}}{\partial t}+\nabla \cdot(\mathbf{v B}-\mathbf{B v}) & =0
\end{aligned}
$$

where $\rho, \mathbf{v}, \mathbf{B}$, and $\mathbf{I}$ are the plasma density, velocity, magnetic field, and unit tensor, respectively. In solar applications, gas pressure is $p=2.3 n_{\mathrm{H}} k_{\mathrm{B}} T$, assuming full ionization and a solar hydrogen to helium abundance $n_{\mathrm{He}} / n_{\mathrm{H}}=0.1$, where $\rho=1.4 n_{\mathrm{H}} m_{p}$ (or the mean molecular weight $\tilde{\mu} \approx 0.6087$ ), while $E=p /(\gamma-1)+\rho v^{2} / 2+B^{2} / 2 \mu_{0}$ is the total energy. Consider $\mathbf{B}=\mathbf{B}_{\mathbf{0}}+\mathbf{B}_{\mathbf{1}}$, where $\mathbf{B}_{\mathbf{0}}$ is a time-independent magnetic field, satisfying $\partial \mathbf{B}_{\mathbf{0}} / \partial t=0$ and $\nabla \cdot \mathbf{B}_{\mathbf{0}}=0$. Equation (18) can be written as

$$
\begin{gathered}
\frac{\partial(\rho \mathbf{v})}{\partial t}+\nabla \cdot\left(\rho \mathbf{v v}+\left(p+\frac{B_{1}^{2}}{2 \mu_{0}}\right) \mathbf{I}-\frac{\mathbf{B}_{\mathbf{1}} \mathbf{B}_{\mathbf{1}}}{\mu_{0}}\right)+ \\
\nabla \cdot\left(\frac{\mathbf{B}_{\mathbf{0}} \cdot \mathbf{B}_{\mathbf{1}}}{\mu_{0}} \mathbf{I}-\frac{\mathbf{B}_{\mathbf{0}} \mathbf{B}_{\mathbf{1}}+\mathbf{B}_{\mathbf{1}} \mathbf{B}_{\mathbf{0}}}{\mu_{0}}\right)=\frac{1}{\mu_{0}}\left(\nabla \times \mathbf{B}_{\mathbf{0}}\right) \times \mathbf{B}_{\mathbf{0}} .
\end{gathered}
$$


Defining $E_{1} \equiv p /(\gamma-1)+\rho v^{2} / 2+B_{1}^{2} /\left(2 \mu_{0}\right)$, then $E=E_{1}+\left(B_{0}^{2}+2 \mathbf{B}_{\mathbf{0}} \cdot \mathbf{B}_{\mathbf{1}}\right) /\left(2 \mu_{0}\right)$ and equation (19) can be written as

$$
\begin{gathered}
\frac{\partial E_{1}}{\partial t}+\frac{\mathbf{B}_{\mathbf{0}}}{\mu_{0}} \cdot \frac{\partial \mathbf{B}_{\mathbf{1}}}{\partial t}+\nabla \cdot\left(E_{1} \mathbf{v}+\left(p+\frac{B_{1}^{2}}{2 \mu_{0}}\right) \mathbf{v}-\frac{\mathbf{B}_{\mathbf{1}}}{\mu_{0}}\left(\mathbf{B}_{\mathbf{1}} \cdot \mathbf{v}\right)\right)+ \\
\nabla \cdot\left(\frac{\mathbf{B}_{\mathbf{0}} \cdot \mathbf{B}_{\mathbf{1}}}{\mu_{0}} \mathbf{v}-\frac{\mathbf{B}_{\mathbf{0}}}{\mu_{0}}\left(\mathbf{B}_{\mathbf{1}} \cdot \mathbf{v}\right)\right)+\nabla \cdot\left(\frac{\mathbf{B}_{\mathbf{0}} \cdot \mathbf{B}}{\mu_{0}} \mathbf{v}-\frac{\mathbf{B}}{\mu_{0}}\left(\mathbf{B}_{\mathbf{0}} \cdot \mathbf{v}\right)\right)=0 .
\end{gathered}
$$

When we take the scalar product of equation (20) with a time-independent $\mathbf{B}_{\mathbf{0}}$, we can use vector manipulations on the right-hand side (RHS) of $\mathbf{B}_{\mathbf{0}} \cdot \partial \mathbf{B}_{\mathbf{1}} / \partial t=\mathbf{B}_{\mathbf{0}} \cdot \nabla \times(\mathbf{v} \times \mathbf{B})$ to obtain

$$
\frac{\mathbf{B}_{\mathbf{0}}}{\mu_{0}} \cdot \frac{\partial \mathbf{B}_{\mathbf{1}}}{\partial t}+\nabla \cdot\left(\frac{\mathbf{B}_{\mathbf{0}} \cdot \mathbf{B}}{\mu_{0}} \mathbf{v}-\frac{\mathbf{B}}{\mu_{0}}\left(\mathbf{B}_{\mathbf{0}} \cdot \mathbf{v}\right)\right)=(\mathbf{v} \times \mathbf{B}) \cdot \frac{1}{\mu_{0}} \nabla \times \mathbf{B}_{\mathbf{0}} .
$$

Inserting equation (23) into equation (22), we arrive at

$$
\begin{gathered}
\frac{\partial E_{1}}{\partial t}+\nabla \cdot\left(E_{1} \mathbf{v}+\left(p+\frac{B_{1}^{2}}{2 \mu_{0}}\right) \mathbf{v}-\frac{\mathbf{B}_{\mathbf{1}}}{\mu_{0}}\left(\mathbf{B}_{\mathbf{1}} \cdot \mathbf{v}\right)\right)+ \\
\nabla \cdot\left(\frac{\mathbf{B}_{\mathbf{0}} \cdot \mathbf{B}_{\mathbf{1}}}{\mu_{0}} \mathbf{v}-\frac{\mathbf{B}_{\mathbf{0}}}{\mu_{0}}\left(\mathbf{B}_{\mathbf{1}} \cdot \mathbf{v}\right)\right)=-(\mathbf{v} \times \mathbf{B}) \cdot \frac{1}{\mu_{0}} \nabla \times \mathbf{B}_{\mathbf{0}} .
\end{gathered}
$$

Therefore, in the ideal MHD system with MFS, we see how a source term appears in the RHS for the momentum equation (21) that quantifies (partly) the Lorentz force $\mathbf{J}_{\mathbf{0}} \times \mathbf{B}_{\mathbf{0}}$, where $\mathbf{J}_{\mathbf{0}}=\nabla \times \mathbf{B}_{\mathbf{0}} / \mu_{0}$, while the energy equation (24) for $E_{1}$ features a RHS written as $\mathbf{E} \cdot \mathbf{J}_{\mathbf{0}}$ for the electric field $\mathbf{E}=-\mathbf{v} \times \mathbf{B}$. The induction equation, for a time-invariant $\mathbf{B}_{\mathbf{0}}$ just writes as before:

$$
\frac{\partial \mathbf{B}_{\mathbf{1}}}{\partial t}+\nabla \cdot\left(\mathbf{v} \mathbf{B}_{\mathbf{1}}-\mathbf{B}_{\mathbf{1}} \mathbf{v}\right)+\nabla \cdot\left(\mathbf{v} \mathbf{B}_{\mathbf{0}}-\mathbf{B}_{\mathbf{0}} \mathbf{v}\right)=\mathbf{0}
$$

For force-free magnetic fields, $\nabla \times \mathbf{B}_{\mathbf{0}}=\alpha \mathbf{B}_{\mathbf{0}}$, where $\alpha$ is constant along one magnetic field line, we have $\mathbf{J}_{\mathbf{0}} \times \mathbf{B}_{\mathbf{0}}=\mathbf{0}$ and $-(\mathbf{v} \times \mathbf{B}) \cdot \mathbf{J}_{\mathbf{0}}=-\left(\mathbf{v} \times \mathbf{B}_{\mathbf{1}}\right) \cdot \mathbf{J}_{\mathbf{0}}$.

For resistive MHD, extra source terms $\nabla \cdot(\mathbf{B} \times \eta \mathbf{J}) / \mu_{0}$ and $-\nabla \times(\eta \mathbf{J})$, are added on the RHS of the energy equation (19) and the induction equation (20), respectively, where $\mathbf{J}=\nabla \times \mathbf{B} / \mu_{0}$. Then the energy equation must account for Ohmic heating and magnetic field diffusion, to be written as

$$
\begin{gathered}
\frac{\partial E_{1}}{\partial t}+\frac{\mathbf{B}_{\mathbf{0}}}{\mu_{0}} \cdot \frac{\partial \mathbf{B}_{\mathbf{1}}}{\partial t}+\nabla \cdot\left(E_{1} \mathbf{v}+\left(p+\frac{B_{1}^{2}}{2 \mu_{0}}\right) \mathbf{v}-\frac{\mathbf{B}_{\mathbf{1}}}{\mu_{0}}\left(\mathbf{B}_{\mathbf{1}} \cdot \mathbf{v}\right)\right)+ \\
\nabla \cdot\left(\frac{\mathbf{B}_{\mathbf{0}} \cdot \mathbf{B}_{\mathbf{1}}}{\mu_{0}} \mathbf{v}-\frac{\mathbf{B}_{\mathbf{0}}}{\mu_{0}}\left(\mathbf{B}_{\mathbf{1}} \cdot \mathbf{v}\right)\right)+\nabla \cdot\left(\frac{\mathbf{B}_{\mathbf{0}} \cdot \mathbf{B}}{\mu_{0}} \mathbf{v}-\frac{\mathbf{B}}{\mu_{0}}\left(\mathbf{B}_{\mathbf{0}} \cdot \mathbf{v}\right)\right) \\
=\eta J^{2}-\frac{1}{\mu_{0}} \mathbf{B}_{\mathbf{0}} \cdot \nabla \times(\eta \mathbf{J})-\frac{1}{\mu_{0}} \mathbf{B}_{\mathbf{1}} \cdot \nabla \times(\eta \mathbf{J}) .
\end{gathered}
$$

The induction equation, extended with resistivity, now gives

$$
\begin{gathered}
\frac{\mathbf{B}_{\mathbf{0}}}{\mu_{0}} \cdot \frac{\partial \mathbf{B}_{\mathbf{1}}}{\partial t}+\nabla \cdot\left(\frac{\mathbf{B}_{\mathbf{0}} \cdot \mathbf{B}}{\mu_{0}} \mathbf{v}-\frac{\mathbf{B}}{\mu_{0}}\left(\mathbf{B}_{\mathbf{0}} \cdot \mathbf{v}\right)\right)= \\
(\mathbf{v} \times \mathbf{B}) \cdot \frac{1}{\mu_{0}} \nabla \times \mathbf{B}_{\mathbf{0}}-\frac{1}{\mu_{0}} \mathbf{B}_{\mathbf{0}} \cdot \nabla \times(\eta \mathbf{J}) .
\end{gathered}
$$

Insert equation (27) to equation (26), and we have the resistive MHD energy equation in MFS representation

$$
\begin{gathered}
\frac{\partial E_{1}}{\partial t}+\nabla \cdot\left(E_{1} \mathbf{v}+\left(p+\frac{B_{1}^{2}}{2 \mu_{0}}\right) \mathbf{v}-\frac{\mathbf{B}_{\mathbf{1}}}{\mu_{0}}\left(\mathbf{B}_{\mathbf{1}} \cdot \mathbf{v}\right)\right)+ \\
\nabla \cdot\left(\frac{\mathbf{B}_{\mathbf{0}} \cdot \mathbf{B}_{\mathbf{1}}}{\mu_{0}} \mathbf{v}-\frac{\mathbf{B}_{\mathbf{0}}}{\mu_{0}}\left(\mathbf{B}_{\mathbf{1}} \cdot \mathbf{v}\right)\right) \\
=-(\mathbf{v} \times \mathbf{B}) \cdot \mathbf{J}_{\mathbf{0}}+\eta J^{2}-\frac{1}{\mu_{0}} \mathbf{B}_{\mathbf{1}} \cdot \nabla \times(\eta \mathbf{J}) .
\end{gathered}
$$

The induction equation as implemented, is then written as

$$
\frac{\partial \mathbf{B}_{\mathbf{1}}}{\partial t}+\nabla \cdot\left(\mathbf{v} \mathbf{B}_{\mathbf{1}}-\mathbf{B}_{\mathbf{1}} \mathbf{v}\right)+\nabla \cdot\left(\mathbf{v} \mathbf{B}_{\mathbf{0}}-\mathbf{B}_{\mathbf{0}} \mathbf{v}\right)=-\nabla \times(\eta \mathbf{J})
$$


In the implementation of the MFS form of the MHD equations in MPI-AMRVAC, we take the following strategies. Each time when creating a new block, a special user subroutine is called to calculate $\mathbf{B}_{\mathbf{0}}$ at cell centers and cell interfaces which is stored in an allocated array, and then $\mathbf{J}_{\mathbf{0}}$ is evaluated and stored at cell centers either numerically or analytically with an optional user subroutine. Note that when the block is deleted from the AMR mesh, these stored values are also lost. The third term in the left hand side of the momentum equation (21), energy equation (24) or (26) and in the induction equation (25) or (29) is added in the flux computation for the corresponding variable, based on pre-determined $\mathbf{B}_{\mathbf{0}}$ magnetic field values at the cell interfaces where fluxes are evaluated. The terms in the right hand sides of all equations are treated as unsplit source terms, which are added in each sub-step of a time integration scheme. We use simple central differencing to evaluate spatial derivatives at cell centers. For example, to compute $\nabla \times(\eta \mathbf{J})$ in the physical region of a block excluding ghost cells, we first compute $\mathbf{J}_{\mathbf{1}}$ in a block region including the first layer of ghost cells, which needs $\mathbf{B}_{\mathbf{1}}$ at the second layer of ghost cells.

\subsection{MFS applications and test problems}

We present three tests and one application to demonstrate the validity and advantage of the MFS technique. All three tests are solved with a finite-volume scheme setup combining the HLL solver (Harten et al. 1983) with Čada's compact third-order limiter (Čada \& Torrilhon 2009) for reconstruction and a three-step Runge-Kutta time integration in a dimensionally unsplit approach. We use a diffusive term in the induction equation to keep the divergence of magnetic field under control (Keppens et al. 2003; van der Holst \& Keppens 2007; Xia et al. 2014). We adopt a constant index of $\gamma=5 / 3$. Unless stated explicitly, we employ dimensionless (code) units everywhere, also making $\mu_{0}=1$.

\subsection{1. $3 D$ force-free magnetic flux rope}

This is a test initialized with a static plasma threaded by a force-free, straight helical magnetic flux rope on a Cartesian grid (see Figure 7). The initial density and gas pressure are uniformly set to unity. The simulation box has a size of 6 by 6 by 6 and is resolved by a $128 \times 128 \times 128$ uniform mesh which is decomposed into blocks of $16 \times 16 \times 16$ cells. The magnetic field is non-potential and nonlinearly force-free, as proposed by Low (Low 1977), and is formulated as

$$
\begin{gathered}
B_{x}=\frac{4 \xi}{1+\xi^{2}} f(y, z), B_{y}=2\left(k z+\frac{1-\xi^{2}}{1+\xi^{2}}\right) f(y, z), B_{z}=-2 k y f(y, z), \\
f(y, z)=B_{a}\left[\frac{4 \xi^{2}}{\left(1+\xi^{2}\right)^{2}}+k^{2} y^{2}+\left(k z+\frac{1-\xi^{2}}{1+\xi^{2}}\right)^{2}\right]^{-1} .
\end{gathered}
$$

We choose free parameters $\xi=1$ and $k=0.5$ to make the axis of the flux rope align with the $x$-axis and set $B_{a}=10$. The electric current of this flux rope is analytically found to be

$$
\begin{gathered}
J_{x}=4 k f(y, z)+4 k B_{a}^{-1} f(y, z)^{2}\left[k^{2} y^{2}+\left(k z+\frac{1-\xi^{2}}{1+\xi^{2}}\right)^{2}\right], \\
J_{y}=\frac{8 \xi k}{1+\xi^{2}} B_{a}^{-1} f(y, z)^{2}\left(k z+\frac{1-\xi^{2}}{1+\xi^{2}}\right), \\
J_{z}=-\frac{8 \xi k}{1+\xi^{2}} B_{a}^{-1} f(y, z)^{2} y .
\end{gathered}
$$

The plasma $\beta$ ranges from 0.006 to 0.027 . The Alfvén crossing time along $y$-axis across the box is 0.377 , determined by the averaged Alfvén speed along $y$-axis. This analytical force-free static equilibrium should remain static for ever. However, due to the finite numerical diffusion of the numerical scheme adopted, the current in the flux rope slowly dissipates when we do not employ the MFS strategy, thereby converting magnetic energy to internal energy and resulting in (small but) non-zero velocities. After 5 (dimensionless code) time units (about 8500 CFL limited timesteps), as shown in Figure 7, the reference run without MFS has up to $20 \%$ increase in temperature near the central axis of the flux rope, while the run with MFS perfectly maintains the initial temperature. Both runs do not have discernible change in magnetic structure.

We quantify the relative changes, $(f(t)-f(0)) / f(0)$, of total magnetic energy, electric current, and internal energy in time for both runs and plot them in Figure 8. In the reference run without MFS, the magnetic energy decreases up to $0.02 \%$, current decreases $0.15 \%$, and internal energy increases $1.8 \%$ at 5 time units. Thus, the dissipation of magnetic energy and current and the resulting increase of internal energy with the employed discretization is significant for long-term runs. However, the run with the MFS strategy keeps the initial equilibrium perfectly and all relative changes are exactly zero. 
(a)

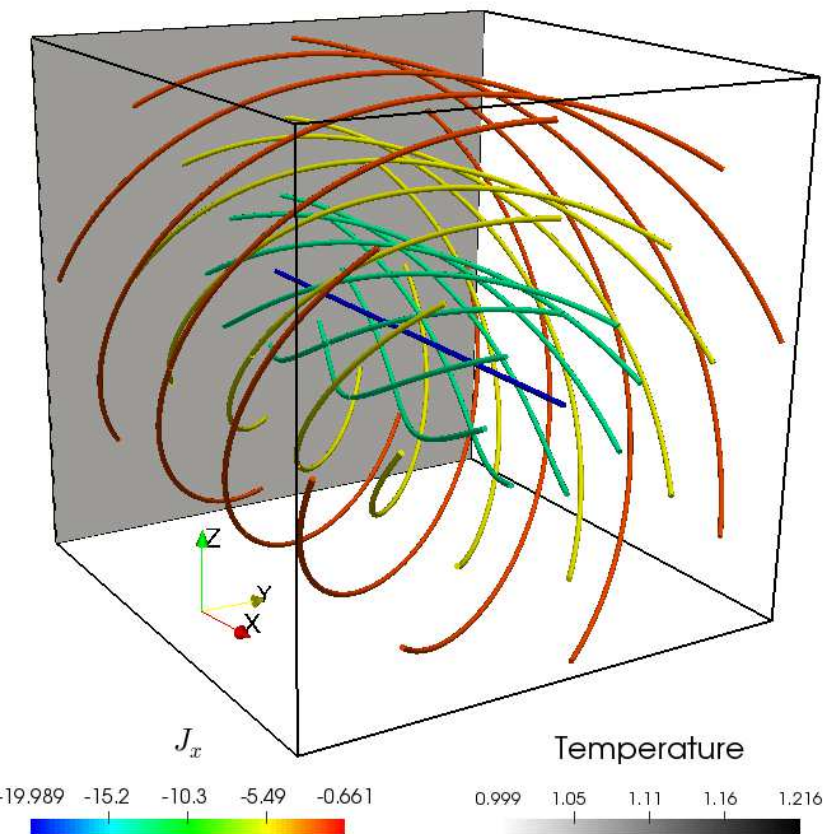

(b)

Time: 5.0

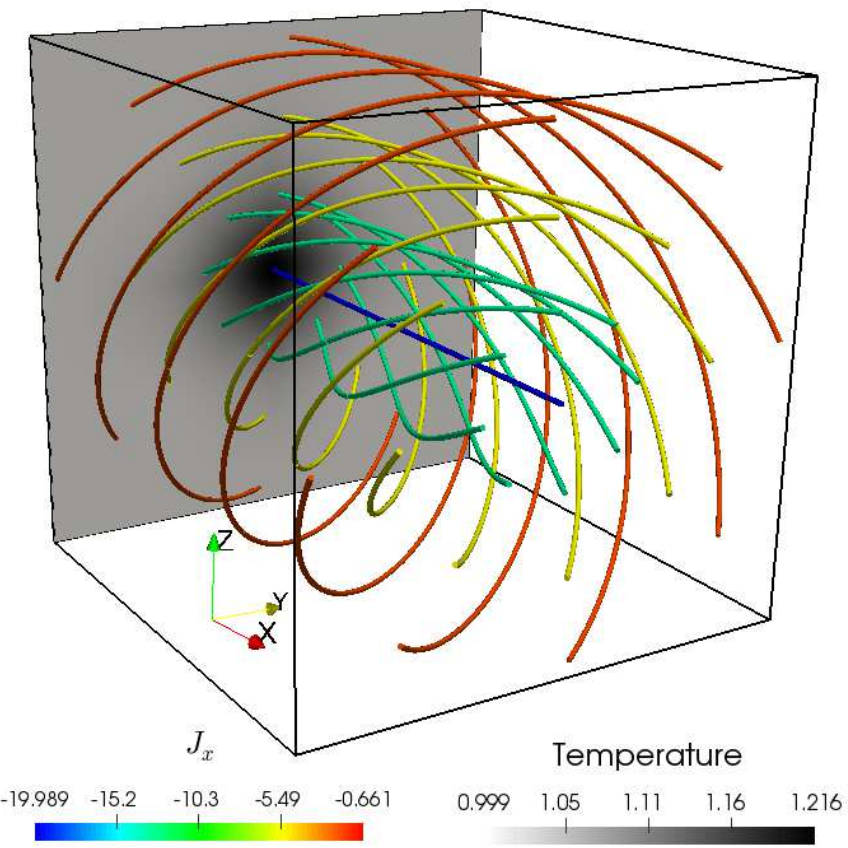

Figure 7. 3D views of the static magnetic flux rope equilibrium after time 5 with (a) and without (b) magnetic field split (MFS), where magnetic field lines are colored by the $x$-component of their current density and the back cross-sections are colored by temperature.

\subsubsection{D non-potential magnetic null-point}

This second test focuses on using the MFS technique in a setup where a magnetic null point is present (where magnetic field locally vanishes) in a $2 \mathrm{D}$ planar configuration. Using a magnetic vector potential $\mathbf{A}=\left[0,0, A_{z}\right]$ with $A_{z}=0.25 B_{c}\left[\left(j_{t}-j_{z}\right)\left(y-y_{c}\right)^{2}-\left(j_{t}+j_{z}\right)\left(x-x_{c}\right)^{2}\right]$, the resulting magnetic field works out to

$$
\begin{gathered}
B_{x}=0.5 B_{c}\left(j_{t}-j_{z}\right)\left(y-y_{c}\right), \\
B_{y}=0.5 B_{c}\left(j_{t}+j_{z}\right)\left(x-x_{c}\right), \\
B_{z}=0 .
\end{gathered}
$$

Its current density is

$$
\begin{gathered}
J_{x}=J_{y}=0, \\
J_{z}=B_{c} j_{z} .
\end{gathered}
$$

This magnetic field has a 2D non-potential null-point in the middle, as seen in the magnetic field lines shown in the left panel of Figure 9. The domain has $x \in[-0.5,0.5]$ and $y \in[0,2]$ and is resolved by a uniform mesh of $128 \times 256$ cells. We take $x_{c}=0$ and $y_{c}=1$ to make the null point appear at the center of the domain. To achieve a static equilibrium, the non-zero Lorentz force is balanced by a pressure gradient force as

$$
\mathbf{J} \times \mathbf{B}-\nabla p=0 .
$$

By solving this, we have the gas pressure as $p(x, y)=B_{c} j_{z} A_{z}+p_{0}$. The initial density is uniformly 1 , and all is static. The arrows in the right panel of Figure 9 only show the direction of the Lorentz force. We choose dimensionless values of $B_{c}=10, p_{0}=7, j_{t}=2.5$, and $j_{z}=1$. The Alfvén crossing time along $y$-axis across the box is about 1 . Plasma $\beta$ ranges from 0.06 at four corners to 8782 near the null point. To test how well the numerical solver maintains this initial static equilibrium, we perform runs with or without MFS. All variables except for velocity are fixed as their initial values in all boundaries, and velocity is made asymmetric about boundary surfaces to ensure zero velocity at all boundaries. 

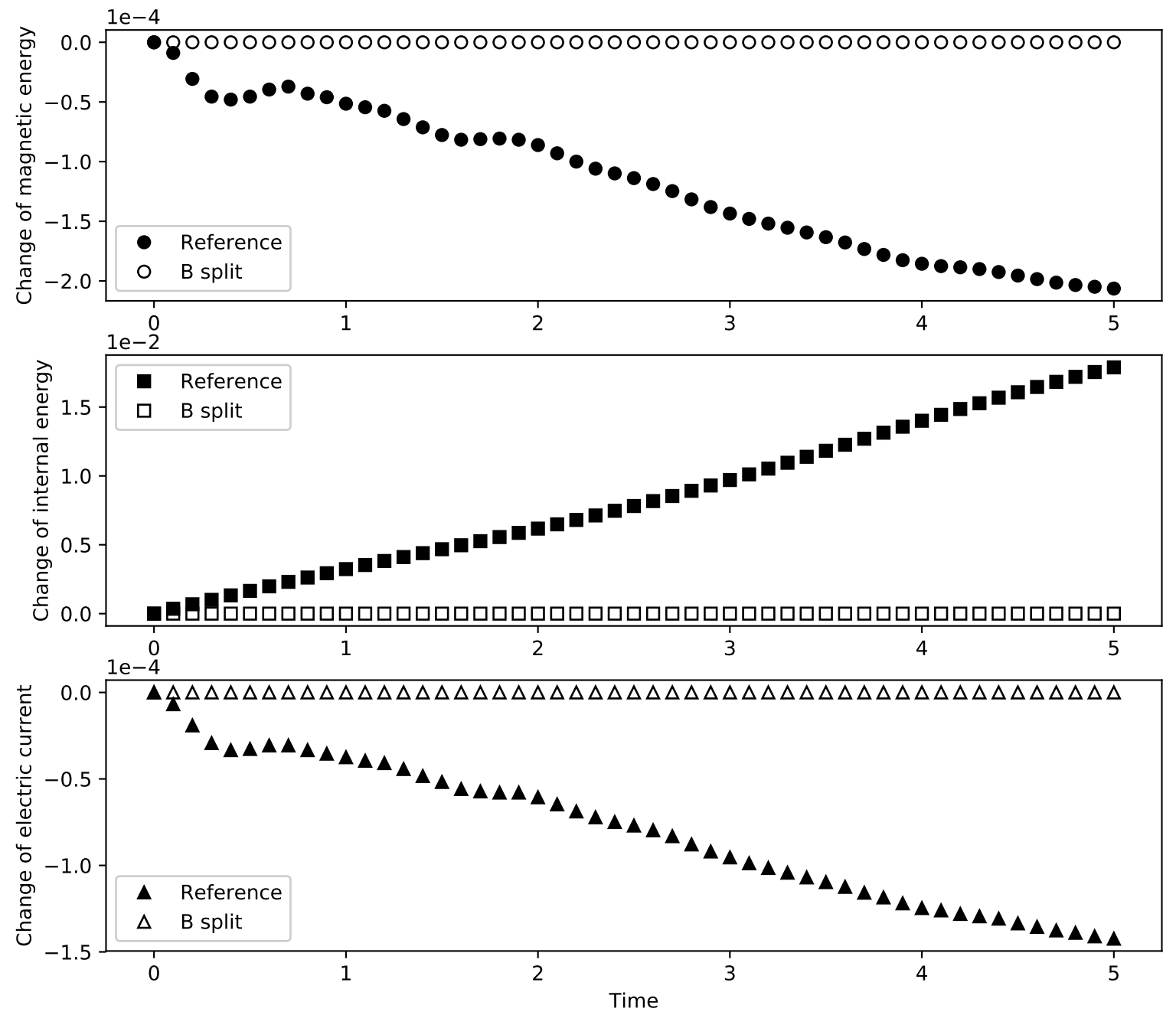

Figure 8. Relative changes of total magnetic energy (top), electric current (middle), and internal energy (bottom) in the run with MFS (B split) shown by hollow symbols and in the reference run shown by filled symbols.

After 10 time units (about 22550 CFL limited time steps) in the MFS run, the density decreases at most $0.038 \%$ in the central horizontal line near the side boundaries and increases $0.02 \%$ in the central vertical line as shown in Figure 9, which corresponds to numerically generated diverging and converging flows to the horizontal and vertical line, respectively, with very small speeds of magnitude at most $2 \times 10^{-4}$, which is only a $6 \times 10^{-3}$ fraction of the local Alfvén speed. This cross-shaped pattern has only four cells in width. The reference run without MFS gives almost the same images as in Figure 9. The MFS run splits off the entire field above as $\mathbf{B}_{\mathbf{0}}$ with its non-zero Lorentz force, and gives essentially the same results as the standard solver for the equilibrium state. We then perform convergence study with increasing grid resolution, and plot kinetic energy, the ratio between kinetic energy and total energy, and magnetic energy normalized by its initial value at 10 time units in Figure 10. Both MFS runs and reference runs without MFS present very similar converging behavior and errors (deviations from the initial equilibrium) decreases with increasing resolution at 5th order of accuracy. Therefore the correctness of MFS technique for non-force-free fields is justified. 

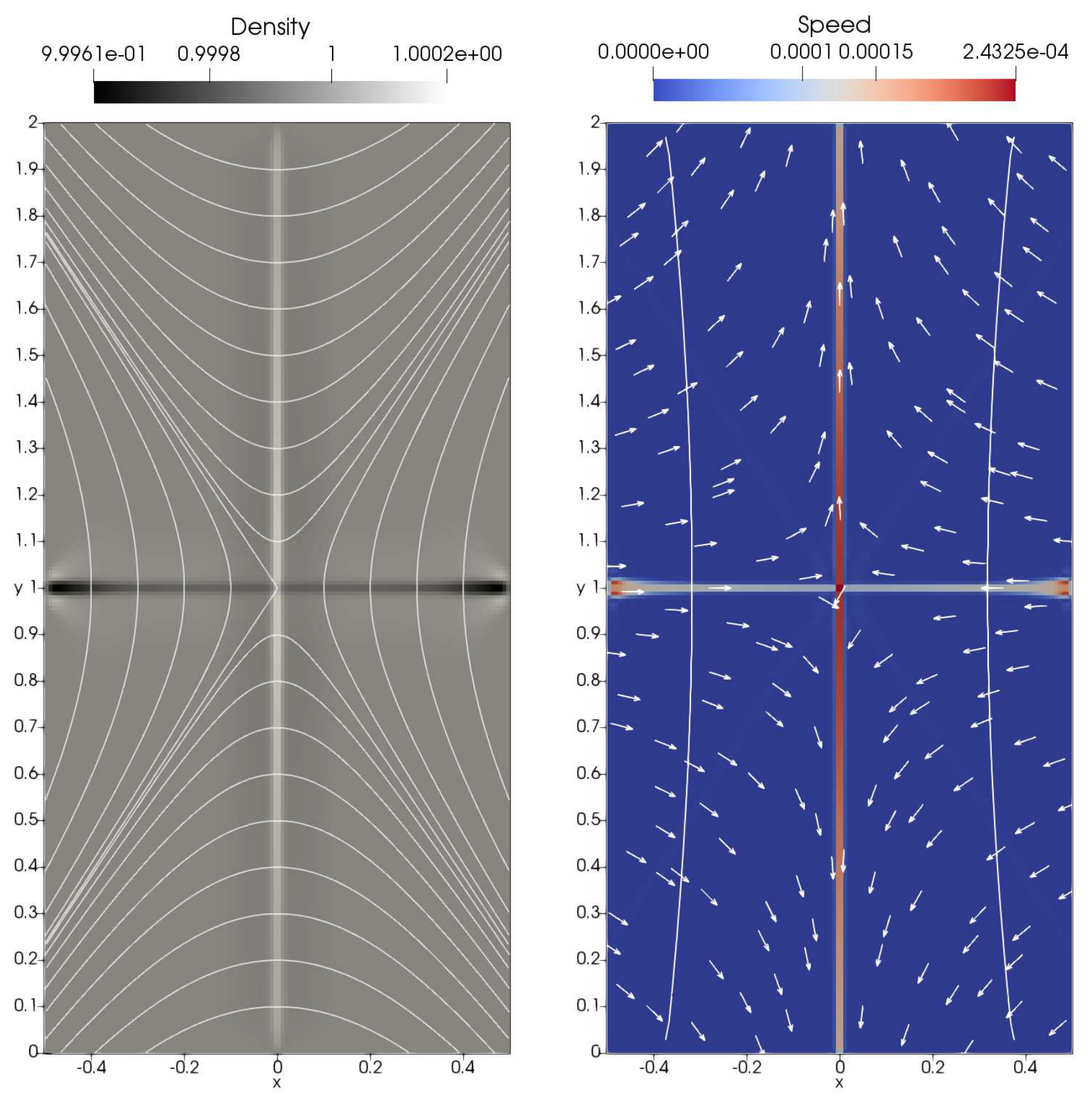

\section{Time: 10.0}

Figure 9. Density map with over-plotted magnetic field lines (left) and speed map with arrows indicating the local directions of Lorentz force (right) and solid lines where plasma $\beta=1$, at 10 time units with MFS.

To test the MFS technique in resistive MHD, we first test dissipation of a straight current sheet with uniform resistivity. The magnetic field changes direction smoothly in the current sheet located at $x=0$ as given by

$$
\begin{gathered}
B_{x}=0, \\
B_{y}=-B_{d} \tanh \left(c_{w} x\right), \\
B_{z}=B_{d} / \cosh \left(c_{w} x\right) .
\end{gathered}
$$



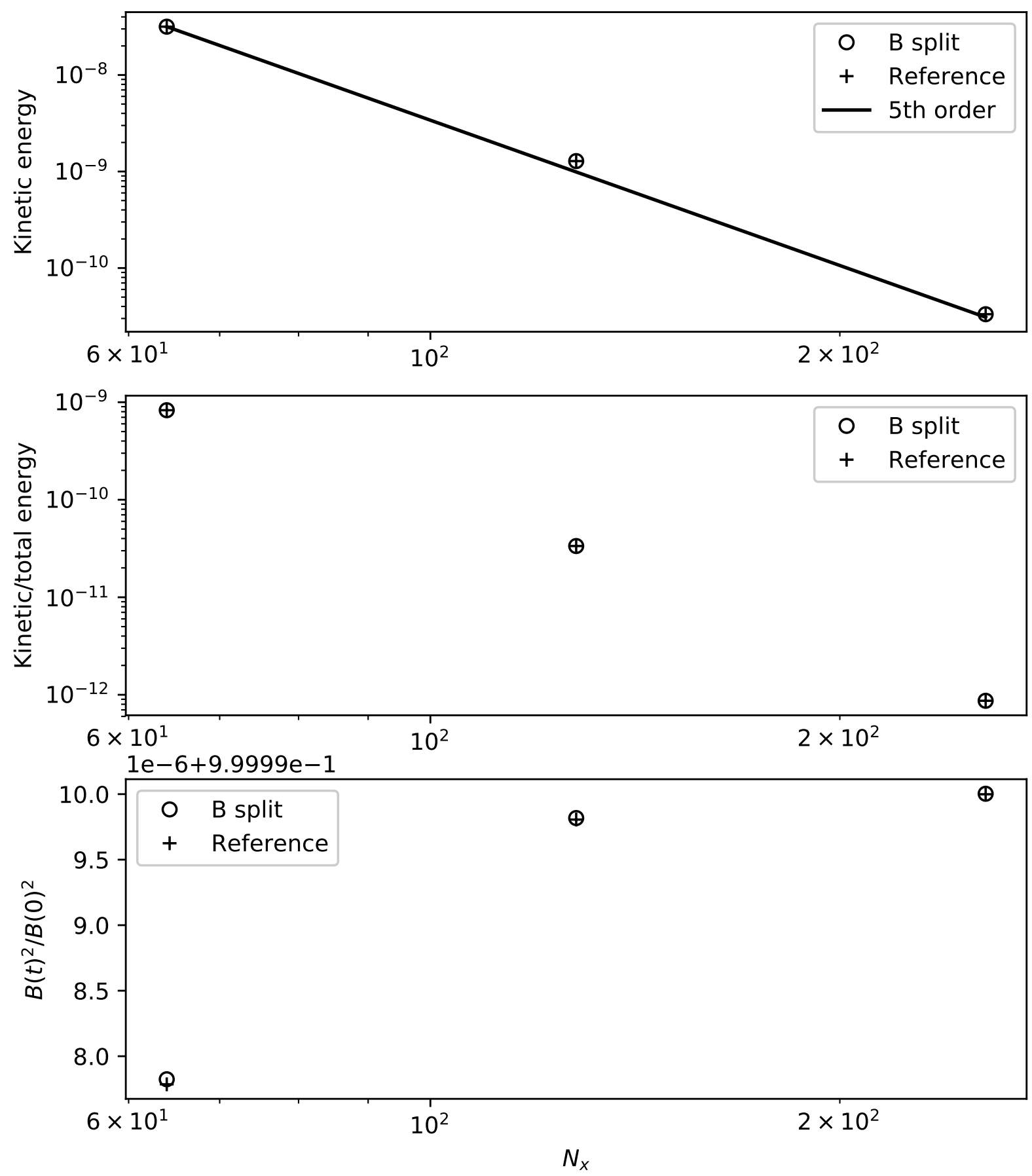

Figure 10. Kinetic energy (top panel), the ratio between kinetic energy and total energy (middle panel), and magnetic energy normalized by its initial value at 10 time units for MFS runs (in circles) and reference runs (in plus) with different grid resolutions $64 \times 128,128 \times 256,256 \times 512$. Note the scale factor for the vertical axis in the bottom panel. 
(a)

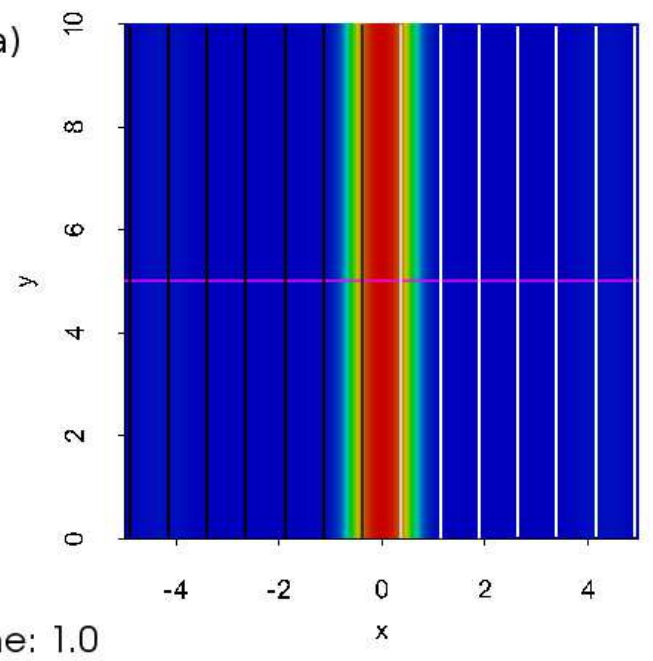

Time: 1.0

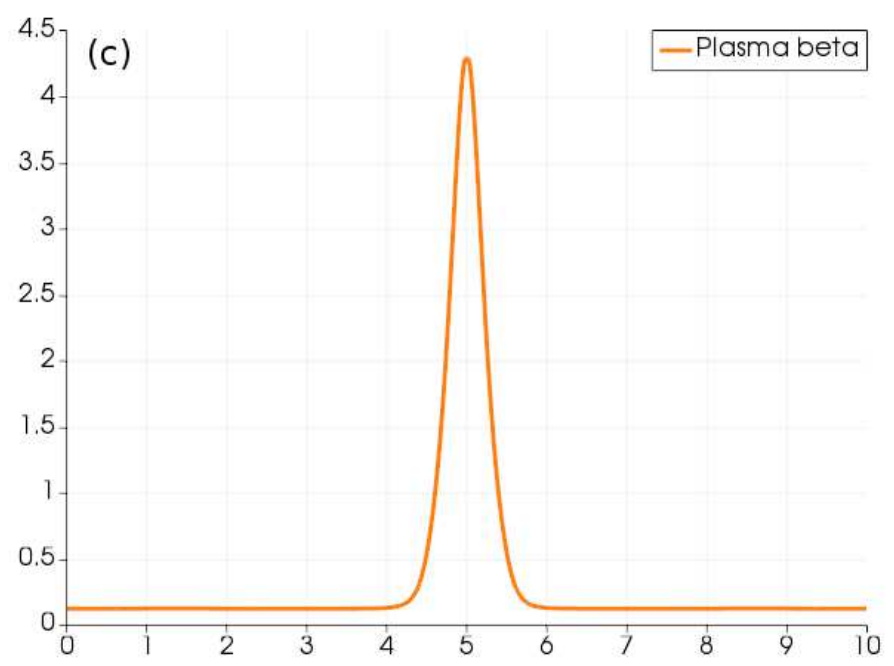

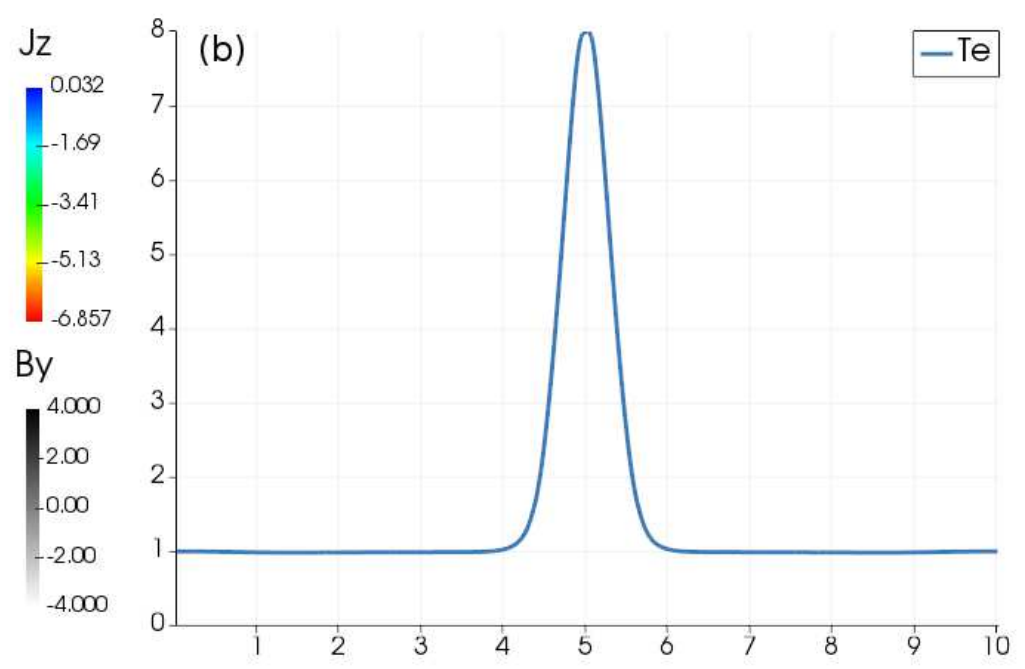

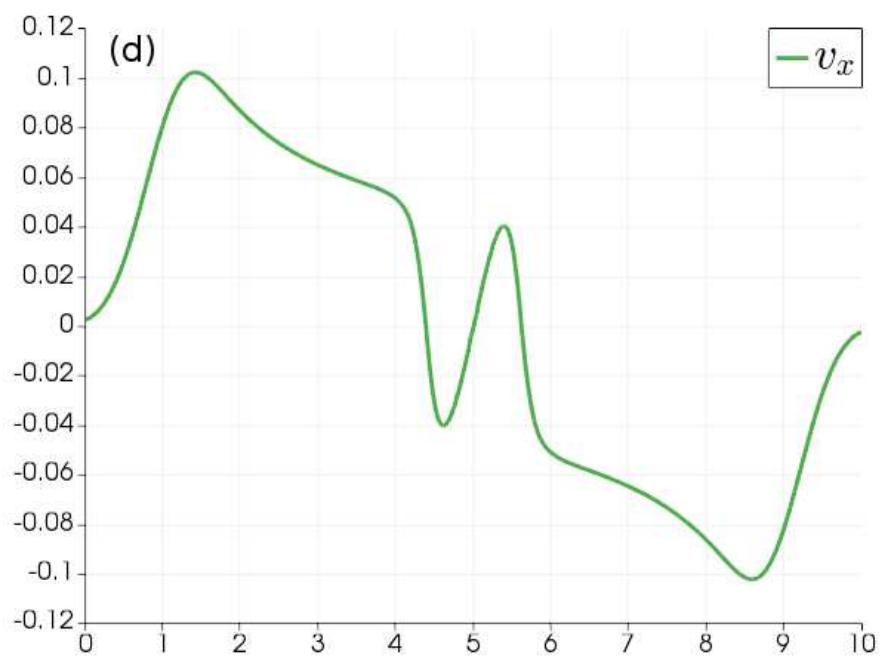

Figure 11. Dissipation of current sheet with MFS at 1 time unit. (a) z-component of electric current over-plotted with magnetic field lines colored by $B_{y}$. A purple line slicing across the current sheet is plotted with its temperature in (b), plasma $\beta$ in (c), and $v_{x}$ in (d).

Its current density is

$$
\begin{gathered}
J_{x}=0, \\
J_{y}=5 B_{d} \tanh \left(c_{w} x\right) / \cosh \left(c_{w} x\right), \\
J_{z}=-5 B_{d} / \cosh ^{2}\left(c_{w} x\right) .
\end{gathered}
$$

In this test, we adopt parameter values $B_{d}=4$ and current sheet width $c_{w}=5$. It is a non-linear force-free field from a previous numerical study of magnetic reconnection of solar flares (Yokoyama \& Shibata 2001). Initial density and gas pressure are uniformly 1 .

We adopt a constant resistivity $\eta$ with a dimensionless value of 0.1 . The simulation box is 10 by 10 discretized on a 4-level AMR mesh with an effective resolution of $512 \times 512$ cells. The average Alfvén crossing time is 2.5. After 1 time unit in a MFS run, the current is dissipated due to the resistivity as shown in panel (a) from Figure 11, presenting the $z$-component of current in rainbow color and magnetic field lines in grey scale. The Ohmic heating increases the temperature in the current sheet from 1 to 8 , as shown in panel (b), with flows towards the current sheet from outside and velocity pointing outward in the heated current sheet as shown in panel (d). Plasma $\beta$ ranges from 0.125 outside the current sheet and 4.2 in the middle of the current sheet. The reference run without MFS gives undistinguishable images as in Figure 11. To exactly compare the two runs, we evaluated the time evolution of magnetic energy, internal energy, and electric current and plot them in circles for MFS run and in triangles for the reference run in Figure 12. The results for the two runs almost overlap and the decrease of magnetic energy corresponds well to the increase of 

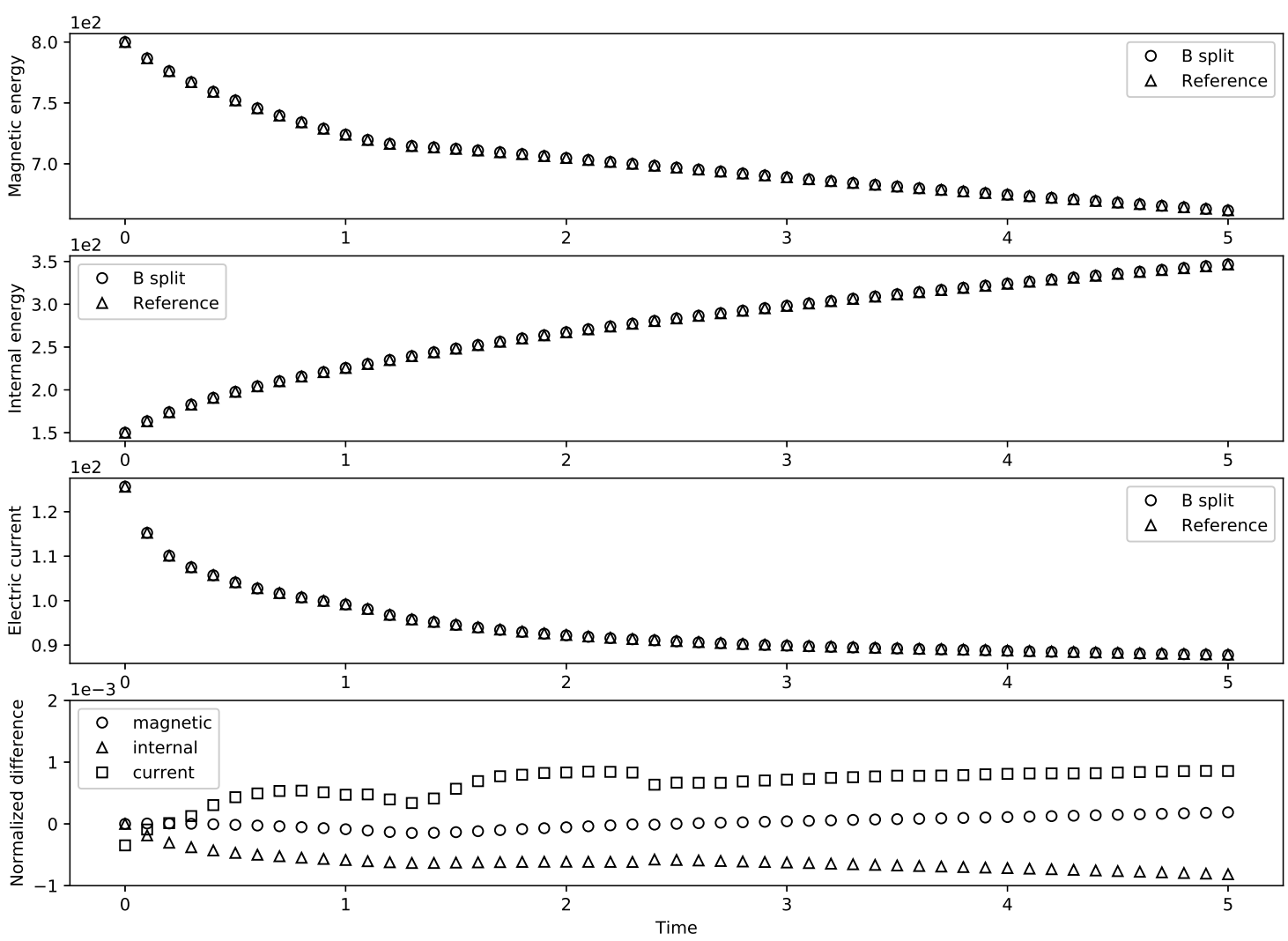

Figure 12. Time evolution of (from top to bottom) magnetic energy, internal energy, and electric current in the case of MFS (in circles) and the non-split reference case (in triangles). The very bottom panel quantifies their relative differences. Note the scale factor in the left top corner at each panel.

internal energy, this time by means of the adopted physical dissipation. We plot the normalized difference between them in the bottom panel, where within $0.1 \%$ differences are found for all three quantities. Therefore, the correctness of MFS technique for resistive MHD is verified.

\subsubsection{Solar flare application with anomalous resistivity}

To show the advantage of MFS technique in fully resistive settings, we present its application on a magnetic reconnection test which extends the simulations of solar flares by Yokoyama \& Shibata (2001) to extremely low- $\beta$, more realistic situations. Here, a run without the MFS technique using our standard MHD solver fails due to negative pressure.

We setup the simulation box in $x \in[-10,10]$ and $y \in[0,20]$ with a four-level AMR mesh, which has an effective resolution of $2048 \times 2048$ and smallest cell size of $97 \mathrm{~km}$. The magnetic field setup is the same current sheet as before, and follows equation (36) with parameters $B_{d}=50$ and $c_{w}=6.667$. Now, a 2D setup is realized with vertically varying initial density and temperature profiles as in Yokoyama \& Shibata (2001), where a hyperbolic tangent function was used to smoothly connect a corona region with a high-density chromosphere. By neglecting solar gravity and set constant gas pressure everywhere, the initial state is in an equilibrium. The current sheet is then first perturbed by an anomalous resistivity $\eta_{1}$ in a small circular region in the current sheet when time $t<0.4$, and afterwards the anomalous resistivity $\eta_{2}$ is switched to a more physics-based value depending on the relative ion-electron drift velocity. The formula of anomalous resistivity $\eta_{1}$ is

$$
\eta_{1}= \begin{cases}\eta_{c}\left[2\left(r / r_{\eta}\right)^{3}-3\left(r / r_{\eta}\right)^{2}+1\right] & \text { if } r \leq r_{\eta}, \\ 0 & \text { if } r>r_{\eta},\end{cases}
$$



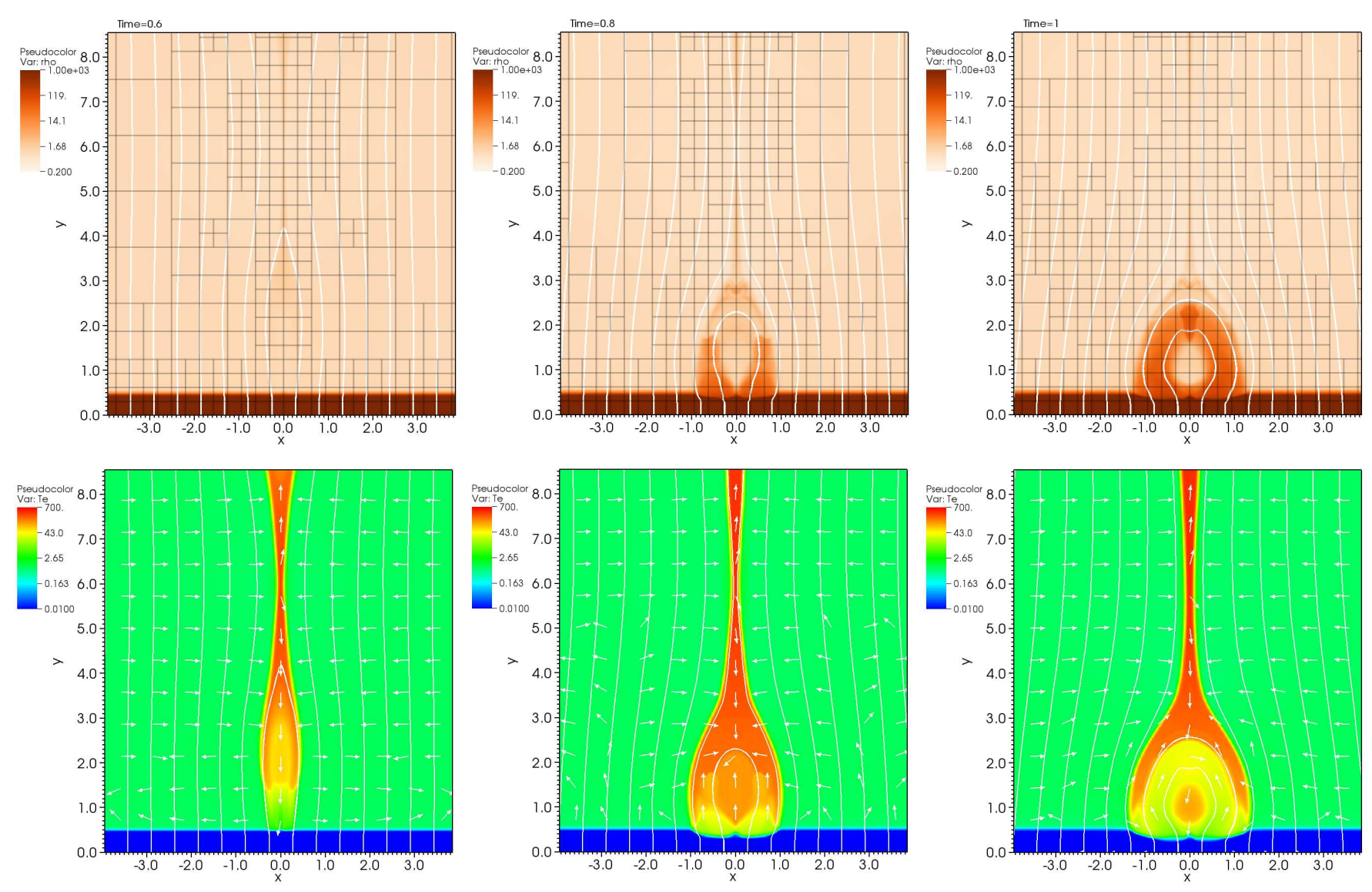

Figure 13. Results of the solar flare simulation. Temporal evolution of density (top) and temperature (bottom) distribution. The arrows show the directions of velocity, and lines show the magnetic field lines integrated from fixed seed points at the bottom. Boarders of AMR blocks are plotted in the density maps. Values in color bars are dimensionless.

where $\eta_{c}=0.002$ is the amplitude, $r=\sqrt{x^{2}+(y-6)^{2}}$ is the distance from the center of the spot at $(x=0, y=6)$, and $r_{\eta}=0.24$ is the radius of the spot. The formula of anomalous resistivity $\eta_{2}$ is

$$
\eta_{2}= \begin{cases}0 & \text { if } v_{d}<v_{c} \\ \alpha_{\eta}\left(v_{d} / v_{c}-1\right) & \text { if } v_{d} \geq v_{c} .\end{cases}
$$

where $v_{d}=\sqrt{J_{x}^{2}+J_{y}^{2}+J_{z}^{2}} /(e n)$ is the relative ion-electron drift velocity ( $e$ is the electric charge of electron and $n$ is number density of plasma), $v_{c}=5 \times 10^{-5}$ is the threshold of the anomalous resistivity, and $\alpha_{\eta}=4 \times 10^{-3}$ is the amplitude.

Continuous boundary conditions are applied for all boundaries and all variables except that $B_{x}=0$ and zero velocity is imposed at the bottom boundary. We basically adopt the same setup and physical parameters as in Yokoyama \& Shibata (2001), except that the magnitude of magnetic field here reaches $100 \mathrm{G}$ with plasma $\beta$ of 0.005 , compared to about $76 \mathrm{G}$ and 0.03 in the original work. We use normalization units of length $L_{u}=10 \mathrm{Mm}$, time $t_{u}=85.87 \mathrm{~s}$, temperature $T_{u}=10^{6} \mathrm{~K}$, number density $n_{u}=10^{9} \mathrm{~cm}^{-3}$, velocity $v_{u}=116.45 \mathrm{~km} \mathrm{~s}^{-1}$, and magnetic field $B_{u}=2 \mathrm{G}$. Since we use different normalization units to normalize the same physical parameters, our dimensionless values are different from Yokoyama's work. We choose a scheme an combining HLL flux with van Leer's slope limiter (van Leer 1974) and two-step time integration. Powell's eight-wave method (Powell et al. 1999) is used to clean divergence of magnetic field. Thermal conduction along magnetic field lines is solved using techniques that are described in details in the following Section 4.

Inspired by Figure 3 in Yokoyama \& Shibata (2001), we show very similar results in Figure 13 which presents snapshots of the density and temperature distribution at times $0.5,0.64$, and 0.75 . The temporal evolution of the magnetic reconnection process and the evaporation in flare loops are reproduced. Flow directions and magnetic field 
lines are also plotted. Magnetic reconnection starts at the center $x=0, y=6$ of the enhanced resistive region, where an X-point is formed. Reconnected field lines with heated plasma are continuously ejected from the X-point to the positive and negative $y$-directions because of the tension force of the reconnected field lines.

\section{ANISOTROPIC THERMAL CONDUCTION}

Thermal conduction in a magnetized plasma is anisotropic with respect to the magnetic field, as the thermal conductivity parallel to magnetic field lines is much larger than the one perpendicular to field lines. This dominantly field-aligned heat flow can be represented by adding the divergence of an anisotropic heat flux to the energy equation. This heat source term can be numerically solved, independently of the MHD equations, using an operator splitting strategy. This means that we ultimately face the challenge to solve an equation due to anisotropic thermal conduction as:

$$
\begin{gathered}
\frac{\partial e}{\partial t}=\nabla \cdot \mathbf{q}, \\
\mathbf{q}=\left(\kappa_{\|}-\kappa_{\perp}\right)(\mathbf{b} \cdot \nabla T) \mathbf{b}+\kappa_{\perp} \nabla T,
\end{gathered}
$$

where $e$ is the internal energy $e=p /(\gamma-1)$ per unit volume, or it is the total energy density $E$ from before. In this equation, $T$ is temperature, $\mathbf{q}$ is the (negative) heat flux vector, $\mathbf{b}=\mathbf{B} / B=\left(b_{x}, b_{y}, b_{z}\right)$ is the unit vector along the magnetic field. The term $\mathbf{b} \cdot \nabla T$ represents the temperature gradient along the magnetic field. $\kappa_{\|}$and $\kappa_{\perp}$ are conductivity coefficients for parallel and perpendicular conduction with respect to the local field direction. In most magnetized astronomical plasma, $\kappa_{\|}$is dominating, for example in the solar corona, where $\kappa_{\perp}$ is about 12 orders of magnitude smaller than $\kappa_{\|}$. Therefore, we can typically ignore physical perpendicular thermal conduction, but we nevertheless implemented both parallel and perpendicular thermal conduction for completeness. Note than when $\kappa_{\perp}=\kappa_{\|}$, the thermal conduction naturally degenerates to isotropic one. In typical astronomical applications, we typically choose the Spitzer conductivity for $\kappa_{\|}=8 \times 10^{-7} T^{5 / 2} \mathrm{erg} \mathrm{cm}^{-1} \mathrm{~s}^{-1} \mathrm{~K}^{-1}$.

\subsection{Discretiziting the heat conduction equation}

To solve the thermal conduction equation (40), various numerical schemes based on centered differencing have been developed. The simplest example is to evaluate gradients directly with second order central differencing; more advanced methods include the asymmetric scheme described in (Parrish \& Stone 2005; Günter et al. 2005) and the symmetric scheme of (Günter et al. 2005). However, these schemes can cause heat to flow low to high temperature if there are strong temperature gradients (Sharma \& Hammett 2007), which is unphysical.

To understand why such effects can occur, consider equation (40). Suppose a finite volume discretization is used in $2 \mathrm{D}$, and that we want to compute the heat flux in the $x$-direction at the cell interface between cell $(i, j)$ and $(i+1, j)$. The term $\left(\kappa_{\|}-\kappa_{\perp}\right)(\mathbf{b} \cdot \nabla T) \mathbf{b}$ is then given by $\left(\kappa_{\|}-\kappa_{\perp}\right)\left(b_{x}^{2} \partial_{x} T+b_{x} b_{y} \partial_{y} T\right)$. The term $b_{x}^{2} \partial_{x} T$ will always be heat flux transported from hot to cold. When $b_{x} b_{y} \partial_{y} T$ has the opposite sign of $b_{x}^{2} \partial_{x} T$ and a larger magnitude, a resulted unphysical heat flux from cold to hot occurs.

Avoiding the above problem, which can lead to negative temperatures, is especially important for astrophysical plasmas with sharp temperature gradients. Examples are the transition region in the solar corona separating the hot corona and the cool chromosphere, or the disk-corona interface in accretion disks. A solution was proposed in Sharma \& Hammett (2007), in which slope limiters were applied to the transverse component of the heat flux. Since the slope limited symmetric scheme was found to be less diffusive than the limited asymmetric scheme, we follow Sharma \& Hammett (2007) and implemented it in our code, with the extra extension to (temperature dependent) Spitzer conductivity and generalized to a 3D setup in Cartesian coordinates. The explicit, conservative finite difference formulation of equation (40) is then

$$
\begin{gathered}
e_{i, j, k}^{n+1}=e_{i, j, k}^{n}+\Delta t\left(\frac{q_{x, i+1 / 2, j, k}^{n}-q_{x, i-1 / 2, j, k}^{n}}{\Delta x}+\right. \\
\left.\frac{q_{y, i, j+1 / 2, k}^{n}-q_{y, i, j-1 / 2, k}^{n}}{\Delta y}+\frac{q_{z, i, j, k+1 / 2}^{n}-q_{y, i, j, k-1 / 2}^{n}}{\Delta z}\right),
\end{gathered}
$$

involving face-centered evaluations of heat flux, e.g. along the $x$-coordinate given by

$$
q_{x}=\kappa b_{x}\left(b_{x} \frac{\partial T}{\partial x}+b_{y} \frac{\partial T}{\partial y}+b_{z} \frac{\partial T}{\partial z}\right)+\kappa_{\perp} \frac{\partial T}{\partial x},
$$

in which $\kappa=\kappa_{\|}-\kappa_{\perp}$. 
Since we use an explicit scheme for time integration, the time step $\Delta t$ must satisfy the condition for numerical stability:

$$
\Delta t \leqslant c_{p} \min \left(\frac{\rho \Delta x^{2}}{(\gamma-1) \kappa_{\|} b_{x}^{2}}, \frac{\rho \Delta y^{2}}{(\gamma-1) \kappa_{\|} b_{y}^{2}}, \frac{\rho \Delta z^{2}}{(\gamma-1) \kappa_{\|} b_{z}^{2}}\right),
$$

where $c_{p}$ is a stability constant taking value of 0.5 and $1 / 3$ for $2 \mathrm{D}$ and $3 \mathrm{D}$, respectively. With increasing grid resolution and/or high thermal conductivity, this limit on $\Delta t$ can be much smaller than the CFL time step limit for solving the ideal MHD equations explicitly. Therefore multiple steps of solving thermal conduction within one step MHD evolution are suggested to alleviate the time step restriction from thermal conduction. In our actual solar coronal applications, we adopt a super timestepping RKL2 scheme proposed by Meyer et al. (2012), where every parabolic update uses an $s$-stage Runge-Kutta scheme with $s$ and its coefficients determined in accord with the 2-term recursion formula for Legendre polynomials. In what follows, we just concentrate on the details to handle a single step for the thermal conduction update.

The first term on the right hand side of expression (44) is discretized as the limited face-centered heat flux:

$$
q_{x x, i+1 / 2, j, k}=\kappa_{i+1 / 2, j, k}\left(\overline{b_{x}^{2} \frac{\partial T}{\partial x}}\right)_{i+1 / 2, j, k},
$$

where the local thermal conduction coefficient is found by first quantifying its value at the cell corners, in $3 \mathrm{D}\left(n_{\mathrm{D}}=3\right)$ found from

$$
\kappa_{i+1 / 2, j+1 / 2, k+1 / 2}=\frac{1}{2^{n_{\mathrm{D}}}} \sum_{n=0}^{1} \sum_{m=0}^{1} \sum_{l=0}^{1} \kappa_{i+l, j+m, k+n},
$$

to then derive the face-centered values as in

$$
\kappa_{i+1 / 2, j, k}=\frac{1}{2^{n_{\mathrm{D}}}-1} \sum_{n=0}^{1} \sum_{m=0}^{1} \kappa_{i+1 / 2, j+1 / 2-m, k+1 / 2-n} .
$$

The face-centered, limited flux expression is given by

$$
\left(\overline{b_{x}^{2} \frac{\partial T}{\partial x}}\right)_{i+1 / 2, j, k}=\frac{1}{2^{n_{\mathrm{D}}}-1} \sum_{n=0}^{1} \sum_{m=0}^{1}\left(b_{x}^{2} \frac{\widetilde{\partial T}}{\partial x}\right)_{i+1 / 2, j+1 / 2-m, k+1 / 2-n},
$$

in which corner values for the magnetic field aligned unit vectors are found as in

$$
b_{x, i+1 / 2, j+1 / 2, k+1 / 2}=\frac{1}{2^{n_{\mathrm{D}}}} \sum_{n=0}^{1} \sum_{m=0}^{1} \sum_{l=0}^{1} b_{x, i+l, j+m, k+n},
$$

while the limited temperature gradient in cell corners is found from

$$
\begin{gathered}
\left.\frac{\widetilde{\partial T}}{\partial x}\right|_{i+1 / 2, j+1 / 2-m, k+1 / 2-n}= \begin{cases}\bar{q} & \text { if } \min q<\bar{q}<\max q, \\
\min q & \text { if } \bar{q} \leqslant \min q, \\
\max q & \text { if } \bar{q} \geqslant \max q,\end{cases} \\
\text { where } \bar{q}=\left.\frac{\partial T}{\partial x}\right|_{i+1 / 2, j+1 / 2-m, k+1 / 2-n}, \\
\quad=\left.\frac{1}{2^{n_{\mathrm{D}}-1}} \sum_{a=0} \sum_{b=0} \frac{\partial T}{\partial x}\right|_{i+1 / 2, j+a, k+b}, \\
\min q=\min \left(\left.\alpha \frac{\partial T}{\partial x}\right|_{i+1 / 2, j, k}, 1 /\left.\alpha \frac{\partial T}{\partial x}\right|_{i+1 / 2, j, k}\right), \\
\text { and finally }\left.\frac{\partial T}{\partial x}\right|_{i+1 / 2, j, k}=\left(T_{i+1, j, k}-T_{i, j, k}\right) / \Delta x .
\end{gathered}
$$


Similar formulae (involving less summations in the averaging) apply at lower dimensionality $n_{\mathrm{D}}$. We choose $\alpha=0.75$ for 2D runs, the same value as used by Sharma \& Hammett (2007), and $\alpha=0.85$ for 3D runs to achieve least numerical diffusion according to our try-and-error numerical tests. Note that in our code, as assumed in the above, the magnetic field components (and other quantities, like temperature) are defined at cell centers, and different formulae would be needed for a staggered grid where magnetic field is defined at cell faces, such as commonly adopted in constrained transport schemes.

The second and the third term of equation (44) are transverse components $q_{x y}$ and $q_{x z}$ of heat conduction, and we illustrate the discretization of the second term here and the third term can be derived similarly:

$$
q_{x y, i+1 / 2, j, k}=\kappa_{i+1 / 2, j, k}\left(b_{x} b_{y} \frac{\overline{\partial T}}{\partial y}\right)_{i+1 / 2, j, k},
$$

where $b_{x, i+1 / 2, j, k}=0.5\left(b_{x, i, j, k}+b_{x, i+1, j, k}\right)$ and $b_{y, i+1 / 2, j, k}$ follow the same formula as equation (48) for $\kappa_{i+1 / 2, j, k}$. The limited, face-centered temperature gradient is evaluated as in

$$
\left.\overline{\frac{\partial T}{\partial y}}\right|_{i+1 / 2, j, k}=L\left(\left.\frac{\partial T}{\partial y}\right|_{i, j, k} ^{L},\left.\frac{\partial T}{\partial y}\right|_{i+1, j, k} ^{L}\right)
$$

in which

$$
\left.\frac{\partial T}{\partial y}\right|_{i, j, k} ^{L}=L\left(\left.\frac{\partial T}{\partial y}\right|_{i, j+1 / 2, k},\left.\frac{\partial T}{\partial y}\right|_{i, j-1 / 2, k}\right)
$$

where $L(a, b)$ is the monotonized central (MC) slope limiter, which is given by

$$
\operatorname{MC}(a, b)=2 \operatorname{sign}(a) \max \{0, \min [|a|, \operatorname{sign}(a) b, \operatorname{sign}(a)(a+b) / 4]\} .
$$

We have tried other slope limiters such as minmod, vanleer, and superbee, but MC limiter gives the best results.

The last term of equation (44) contributes to perpendicular thermal conduction and its discretized form computes the averaged face value of $\kappa_{\perp, i+1 / 2, j, k}$ using the same form as equation (48) and the averaged face value of the temperature gradient along $x$ as in equation (49) (and the equations that follow it), but without the factors $b_{x}^{2}$.

Since thermal conduction in dilute plasmas is caused by the movement of electrons, a larger thermal conduction flux implies faster electrons. Once the electron speed becomes comparable to the sound speed, plasma instabilities prevent the electrons from flowing faster and thermal conduction must saturate (Cowie \& McKee 1977). We adopt the following formula from Cowie \& McKee (1977) for a saturated thermal flux:

$$
q_{\mathrm{sat}}=5 \phi \rho c_{\mathrm{iso}}^{3},
$$

where $c_{\text {iso }}$ is the isothermal sound speed and the factor $\phi=1.1$ when the electron and ion temperatures are equal. We evaluate the projected saturated thermal flux at each cell face based on the averaged density and temperature there, and set it as the upper limit for the total thermal flux through that cell face, namely, $\left|q_{x, i+1 / 2, j, k}\right| \leqslant q_{\mathrm{sat}, i+1 / 2, j, k}\left|b_{x, i+1 / 2, j, k}\right|$.

We implement the methods mentioned above in a thermal conduction module with source code written in the dimension-independent LASY way. Thus the same compact source code can be translated to pure Fortran code in 1D, $2 \mathrm{D}$, or $3 \mathrm{D}$ before compilation.

\subsection{Anisotropic heat conduction test problems: $2 D$ to $3 D$}

A 2D circular ring diffusion test problem was proposed by (Parrish \& Stone 2005) and became a standard test for anisotropic thermal conduction (Sharma \& Hammett 2007; Meyer et al. 2012). It initializes a hot patch in circular magnetic field lines in a $[-1,1] \times[-1,1]$ Cartesian box with temperatures

$$
T= \begin{cases}12 & \text { if } 0.5<r<0.7 \text { and } \frac{11}{12} \pi<\theta<\frac{13}{12} \pi \\ 10 & \text { otherwise }\end{cases}
$$

where $r=\sqrt{x^{2}+y^{2}}$ and $\tan \theta=y / x$. Density is set to 1 . We create circular magnetic field lines centered at the origin by the formula:

$$
B_{x}=10^{-5} \cos (\theta+\pi / 2) / r, \quad B_{y}=10^{-5} \sin (\theta+\pi / 2) / r,
$$

which is generated by a infinite line current perpendicular to the plane through the origin. The parallel thermal conductivity is constant and set to $\kappa_{\|}=0.01$ and no explicit perpendicular thermal conduction is considered. Continuous 
(a)

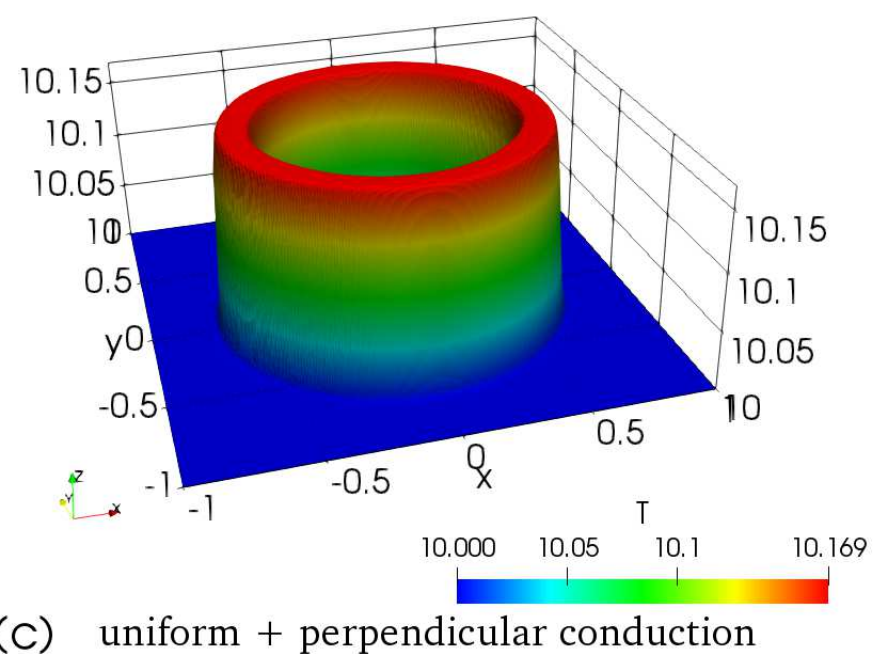

(c)

uniform + perpendicular conduction

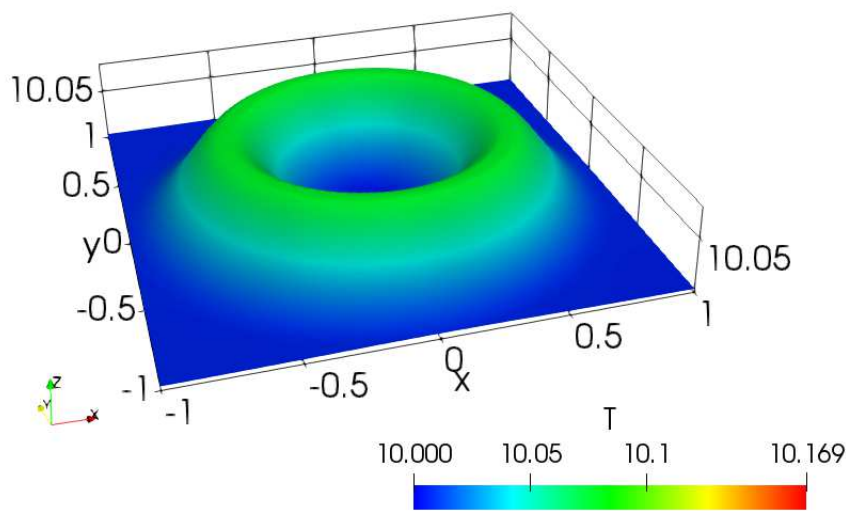

(b)

AMR

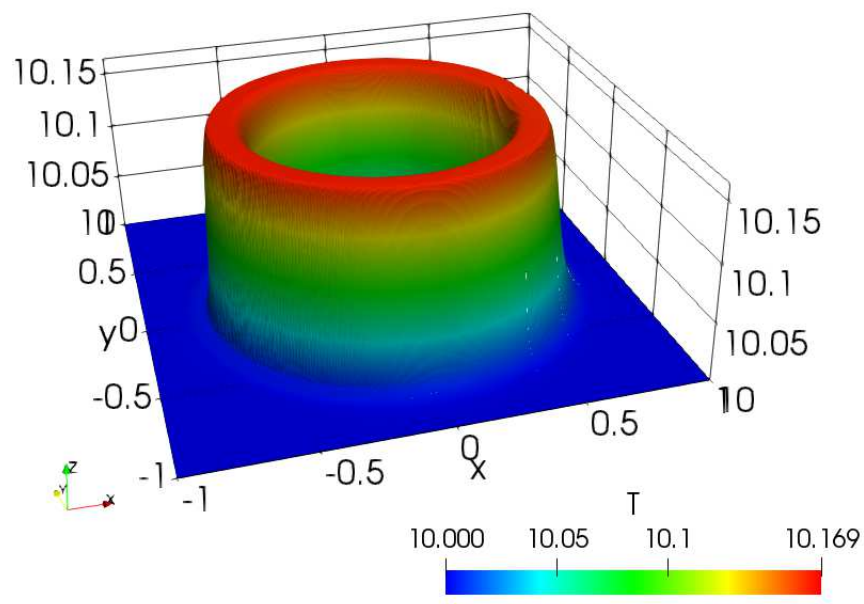

(d)

AMR without conservation fix

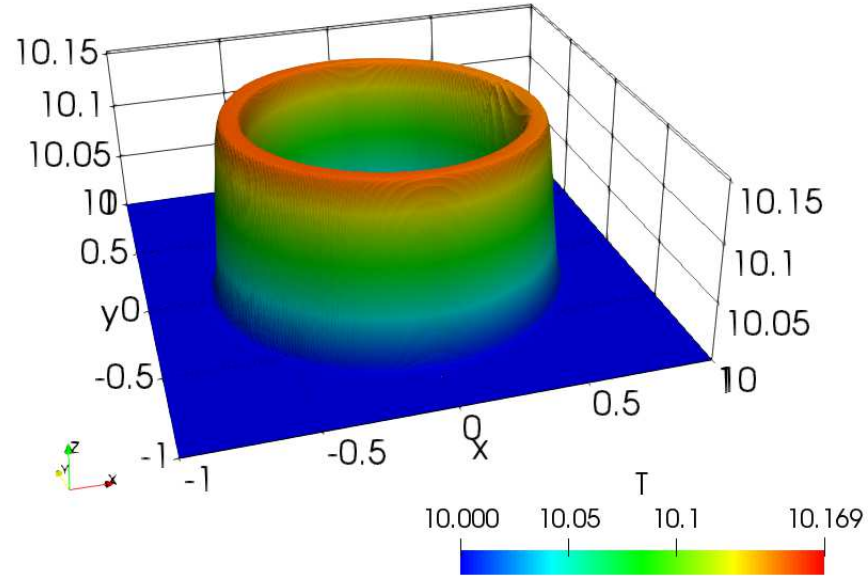

Figure 14. Temperature at 400 time units for four cases initialized with the ring diffusion problem. (a) on uniform $200 \times$ 200 grid; (b) on AMR grid with $200 \times 200$ effective resolution; (c) the same condition as (a) except that perpendicular conduction with $\kappa_{\perp}=0.00005$ is included; (d) the same condition as (b) except for excluding conservation fix.

boundary condition is used for all variable at all boundaries. We only evolve the anisotropic thermal heat conduction equation without solving the MHD equations, until 400 time units. Ideally, the diffusion of temperature should only occur in a ring $0.5<r<0.7$ and eventually result in a uniform temperature of 10.1667 inside the ring with unperturbed temperature of 10 outside the ring. But perpendicular numerical diffusion may cause some cross-field thermal conduction and can smooth sharp temperature gradients at borders of this ring.

The end state of a run (a) on a uniform $200 \times 200$ grid is shown in Figure 14(a). Temperature is high and nearly uniform inside the ring, with a constant background temperature of 10. The maximal and minimal temperature of the end state are 10.1687 and 10, respectively. No temperature overshooting is found near sharp temperature gradient regions and monotonicity property is preserved. The final temperature distribution agrees with previous findings (see the fourth panel in Figure 6 of Sharma \& Hammett 2007). Since the exact solution of this problem is a uniform temperature of 10.1667 in the ring and 10 outside it, we can evaluate the $L_{1}, L_{2}$, and $L_{\infty}$ norm errors for four runs with increasing resolutions (see table 1 ). The $L_{1}$ norm converges with a rate of 1.4 and $L_{2}$ norm converges with a rate of 0.8. The maximal temperature is getting closer to the correct solution with increasing resolution and the minimal temperature stays at the correct value of 10. A rough estimate for the time averaged perpendicular numerical diffusion (conductivity) $\kappa_{\perp, \text { num }}$ and its ratio to $\kappa_{\|}=0.01$ are calculated following the same method in Sharma \& Hammett (2007), and reported in the last column of table 1. Note in particular that we can get this ratio from order $10^{-3}$ down 
Table 1. Accuracy measures of the ring diffusion tests on uniform grids

\begin{tabular}{ccccccc}
\hline Resolution & $L_{1}$ error & $L_{2}$ error & $L_{\infty}$ error & $T_{\max }$ & $T_{\min }$ & $\kappa_{\perp, \text { num }} / \kappa_{\|}$ \\
\hline $50 \times 50$ & 0.03037 & 0.04705 & 0.08617 & 10.0842 & 10 & $4.345 \times 10^{-3}$ \\
$100 \times 100$ & 0.01338 & 0.02704 & 0.11654 & 10.1355 & 10 & $5.145 \times 10^{-4}$ \\
$200 \times 200$ & 0.00521 & 0.01592 & 0.08683 & 10.1663 & 10 & $1.050 \times 10^{-4}$ \\
$400 \times 400$ & 0.00320 & 0.01224 & 0.08758 & 10.1681 & 10 & $3.353 \times 10^{-5}$ \\
\hline
\end{tabular}

to order $10^{-5}$ on sufficiently large grids, so that we can add physical perpendicular thermal conduction coefficients that exceed these values.

In order to use the thermal conduction on a AMR grid, refluxing operations are needed to ensure flux conservation across fine-coarse interfaces. We store the thermal fluxes of blocks with finer or coarser neighbor blocks, and send thermal fluxes at fine-coarse interfaces from finer blocks to coarser blocks. This is standard practice for ensuring conservation in finite volume AMR computations, and our strategy in terms of parallel communication patterns involved is found in section 3.4 of Keppens et al. (2012). Figure 14(b) shows the end state of run (b) on an AMR grid with three levels and an effective resolution of $200 \times 200$. We use dynamic AMR, with the refine-triggering based on total energy using a Löhner type estimator (Lohner 1987; Keppens et al. 2012) and set a refine threshold of 0.08. Comparing to the result of the run in panel (a) on a uniform grid with the same resolution, run (b) gives comparable results. The AMR advantage is in the fact that it uses about $20 \%$ less computational time. Some minor quality degeneracy is present, with less sharp boarders of the ring segment at the far end from the initial hot segment and a lower maximal temperature of 10.1638 . This is likely caused by the larger numerical diffusion associated with the coarser grids at the far end. To prove the importance of the conservation fix, we show a run (d) with the same setup as run (b), except that it has no conservation fix, which ends up with lower temperature in the ring and stronger diffusion at its boarders, as shown in Figure 14(d). To test perpendicular thermal conduction, we perform test run (c) with a constant $\kappa_{\perp}=5 \times 10^{-5}=5 \times 10^{-3} \kappa_{\|}$and other setups the same as run (a). The final hot ring is significantly smoothed by perpendicular thermal conduction.

We generalize the $2 \mathrm{D}$ ring diffusion test to a $3 \mathrm{D}$ version. In a $3 \mathrm{D}[-1,1]^{3}$ Cartesian box, we rotate the magnetic field loops, from a position at first in $x$-y planes, around $x$-axis 45 degrees and then rotate around $z$-axis 45 degrees, to make the magnetic field direction misaligned with the coordinates. Therefore, the thermal flux along magnetic field lines must include contributions from all three dimensions. An initial 3D hot patch is set to have the same extension in the plane of the magnetic loop as in the $2 \mathrm{D}$ case and an extension of 0.4 in the direction perpendicular to this plane. We run the test on a uniform $200 \times 200 \times 200$ mesh until 400 time units. As shown in Figure 15(a), the 3D isosurface of temperature at 10.16 represents a ring shape. A slice through the middle plane of the ring structure is shown in a warped view and colored by temperature in Figure 15(b), and is comparable with the previous 2D results. The maximal temperature in the hot ring of the slice is 10.1774 and the thickness of the hot ring is $10 \%$ larger than in the corresponding 2D tests. A translucent slice cutting through the ring in panel (a) shows, in panel (c), that its cross section has a rounded square shape, which is caused by large numerical diffusion at its corner edges. To test the 3D thermal conduction on a AMR mesh, we run the same 3D test problem on a 3-level AMR mesh with base-level resolution of $50 \times 50 \times 50$, which has the same effective resolution as the previous test on a uniform mesh. The result is very similar to the uniform-mesh version with maximal temperature of 10.1727 , while the run consumes $45 \%$ less computational time than the uniform-mesh run.

To check the consistency between the 3D implementation and the 2D one, we set up a test of 2D ring diffusion problem in a 3D simulation. We set the magnetic field loops in $x-y$ planes and the initial hot patch has the same extension in $x$ and $y$ directions as in the 2D setup and extends uniformly through the box in $z$ direction. Periodic boundary condiction is used at $z$ boundaries and continuous boundary condiction is adopted in $x$ and $y$ boundaries. The simulation box has a size of 1 by 1 by 0.1 on a $200 \times 200 \times 20$ mesh. The temperature distribution is invariant in $z$ direction and its $x-y$ plane slice is very close to the $2 \mathrm{D}$ result with the same resolution at 400 time units. The maximum and miminum temperatures are the same. The temperature transition region is about $4 \%$ broader in the $3 \mathrm{D}$ run. Therefore, the $3 \mathrm{D}$ implementation is consistent with the $2 \mathrm{D}$ one.

\section{PERFORMANCE AND SCALING}

In the previous version of MPI-AMRVAC, the root-level blocks were connected with a simple dimension-by-dimension incremental order curve, while a Morton-ordered space filling curve was recursively applied from level 2 onwards, refining root level blocks, to the leaf levels (Keppens et al. 2012). In MPI-AMRVAC 2.0, we also connect the root-level 

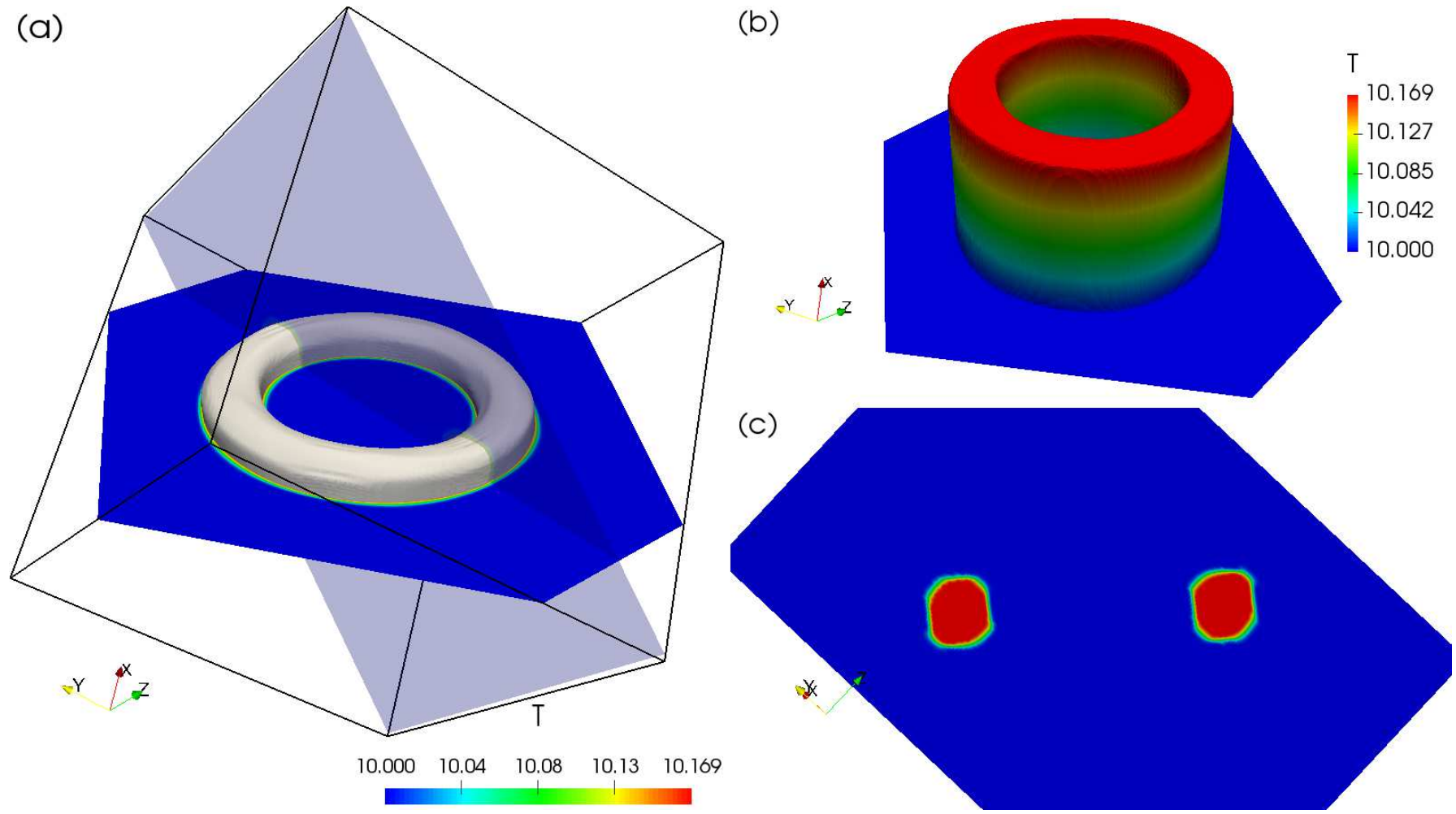

Figure 15. Temperature at 400 time units in a 3D case initialized with the ring diffusion problem on a uniform $200 \times 200 \times 200$ grid. (a) isosurface of temperature at 10.16 and a plane slicing through the middle of the hot patch in the 3D box; (b) 3D view of the 2D slice plane in (a), warped and colored by temperature; (c) temperature on the translucent slice in (a).

blocks with a Morton-ordered space filling curve, as illustrated in Figure 16, to improve data locality and minimize times of inter-processor communications. Since a pure Morton curve connects nodes arranged in a square shape in $2 \mathrm{D}$ or in a cubic shape in 3D which have the same even number of node blocks along each dimension, we can not directly apply it to a domain with nodes arranged in a non-square shape or with odd number of node blocks. In a true rectangular case, e.g. $5 \times 2$ in Figure 16, we first extend the rectangular domain to a square one, namely, $6 \times 6$, apply the standard Morton curve on it, and remove nodes that lay outside of the rectangular domain. To remove a node on a space filling curve, we simply delete the node and subtract one from the numbers of all nodes behind it. In addition to that, we further reduce communication by skipping the communication and update of corner cells, if they are not needed in a certain physics solver. Optimizations include speeding up the flux calculation, avoid using split source terms which need an extra ghost cells update by communication, and minimizing the conversion between primitive and conservative variables, (e.g., provide both primitive and conservative left and right state at cell interfaces after reconstruction for flux evaluation).

With this new block ordering, we present two sets of tests to demonstrate strong scaling of MPI-AMRVAC on Breniac, the Flemish Tier-1 supercomputer. The first one is solving a 3D blast wave problem in ideal MHD physics. The simulation box is of size 2 by 2 by 2 on a uniform mesh of $512^{3}$ cells. The mesh is decomposed into $32^{3}$ blocks with each block of $16^{3}$ cells. Initially, gas pressure is 100 inside a central ball with radius of 0.2 and 1 outside, density is uniformly 1 , magnetic field is uniform with $B_{x}=B_{y}=B_{z}=1$, and velocity is zero. We use the same schemes as explained in the beginning of Section 3.2, namely, HLL flux, Čada's compact third-order limiter, the three-step Runge-Kutta time integration, and the diffusive approach to clean divergence of magnetic field. To do strong scaling test, we run the same test with increasing number of cores, starting from 28 cores to 3584 cores. We plot speed as cells updated per second and efficiency of speed up in Figure 17, where the black line shows ideal scaling with 100\% efficiency. The runs with the previous code version MPI-AMRVAC 1.0, plotted in empty triangle, stop speeding up after 1729 cores, while the new MPI-AMRVAC 2.0, plotted in dots, shows much better scaling. When running on 3584 cores (37449 cell per core), the test with MPI-AMRVAC 2.0 still has $54 \%$ speed-up efficiency with respect to the speed of 28 cores. Note that each data point reported here is an average result of three repeated runs. 


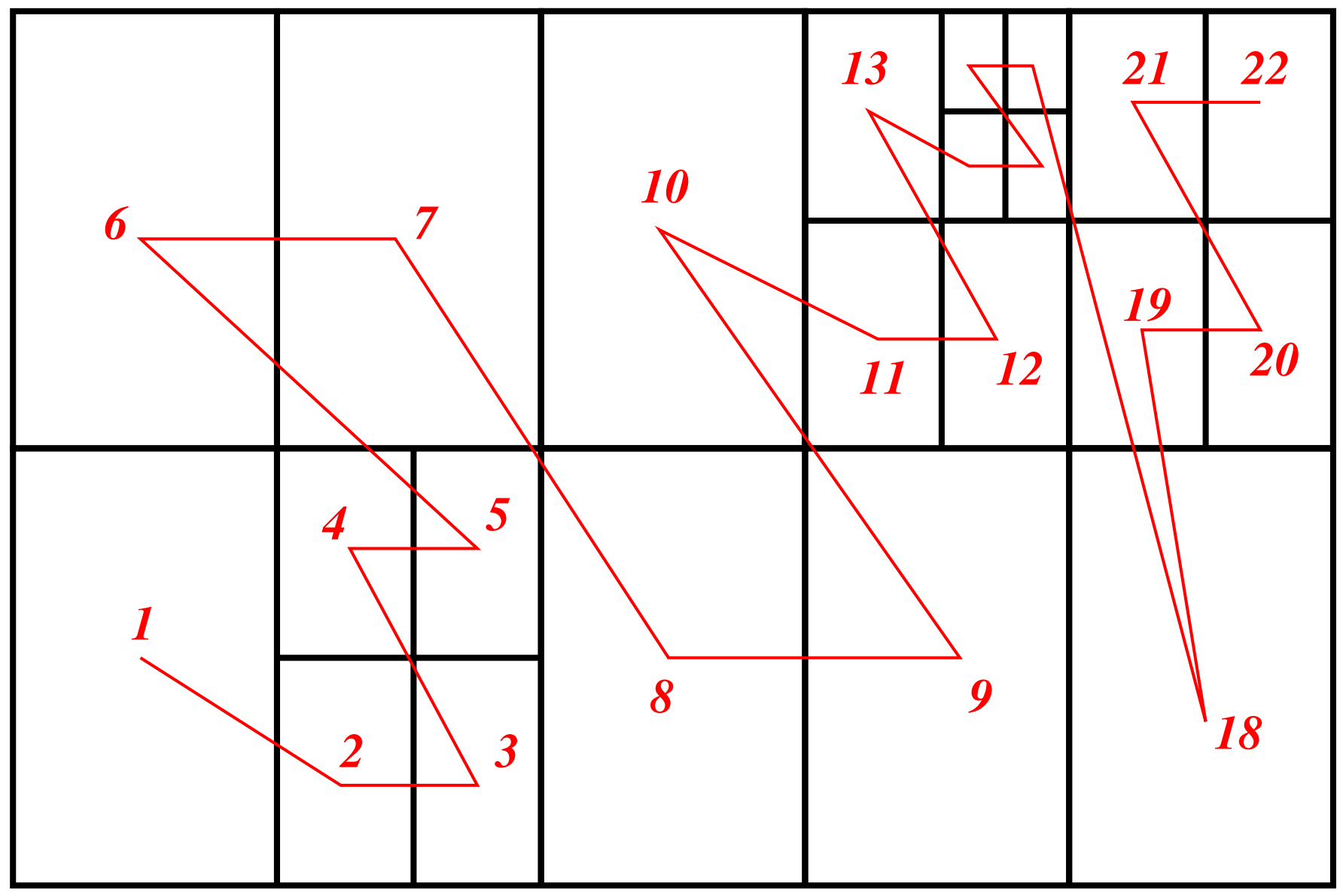

Figure 16. Morton ordered curve illustrated for a rectangular domain with $5 \times 2$ root level blocks.

The second test comes from an actual solar application, where we have done a 3D coronal rain dynamics simulation (Xia et al. 2017), which solves MHD coupled with parameterized heating, optically thin radiative cooling, and anisotropic thermal conduction as described in Section 4. We used an AMR mesh to resolve high-density, lowtemperature coronal rain blobs when they form and fall down in a magnetic arcade (Xia et al. 2017). The AMR mesh is composed of $12^{3}$-cell (excluding ghost cells) blocks organized in 5 AMR levels. To do this strong scaling test, we take a snapshot (at time $46.3 \mathrm{~min}$ ) from this coronal rain simulation, restart the simulation from that moment, and run for 10 time steps, with once data output in the end. About 46.4 million cells in 26652 blocks are evolved. AMR mesh is reevaluated for (de)refinement every 3 time steps, although in the end the mesh structure and number of blocks does not change during the test. We use the same schemes as in the first test and the MFS technique mentioned in Section 3. The results of a strong scaling test are shown in Figure 18. More than $60 \%$ efficiency is obtained when using up to about 2000 cores, with more than $5 \times 10^{4}$ cells per core.

\section{GENERIC FRAMEWORK IMPROVEMENTS}

MPI-AMRVAC has been in development since early this century (Nool \& Keppens 2002), and many scientists contributed code to it. These contributions usually aimed to enable new research, for example by adding numerical methods or extending physics models. In the most recent restructuring effort, we modernized the underlying framework, which will hopefully help in maintaining and developing the framework in the decades to come. In this section, we describe improvements we made in this regard.

\subsection{Modernization of the framework}

MPI-AMRVAC is written in Fortran extended by the LASY syntax (Tóth 1997). After source files have been translated by a LASY preprocessor, they can be compiled by a standard Fortran compiler. The main goal of the LASY syntax is to be able to write dimension-independent code. However, the LASY syntax was also used to for example:

- (optionally) include other source files, 

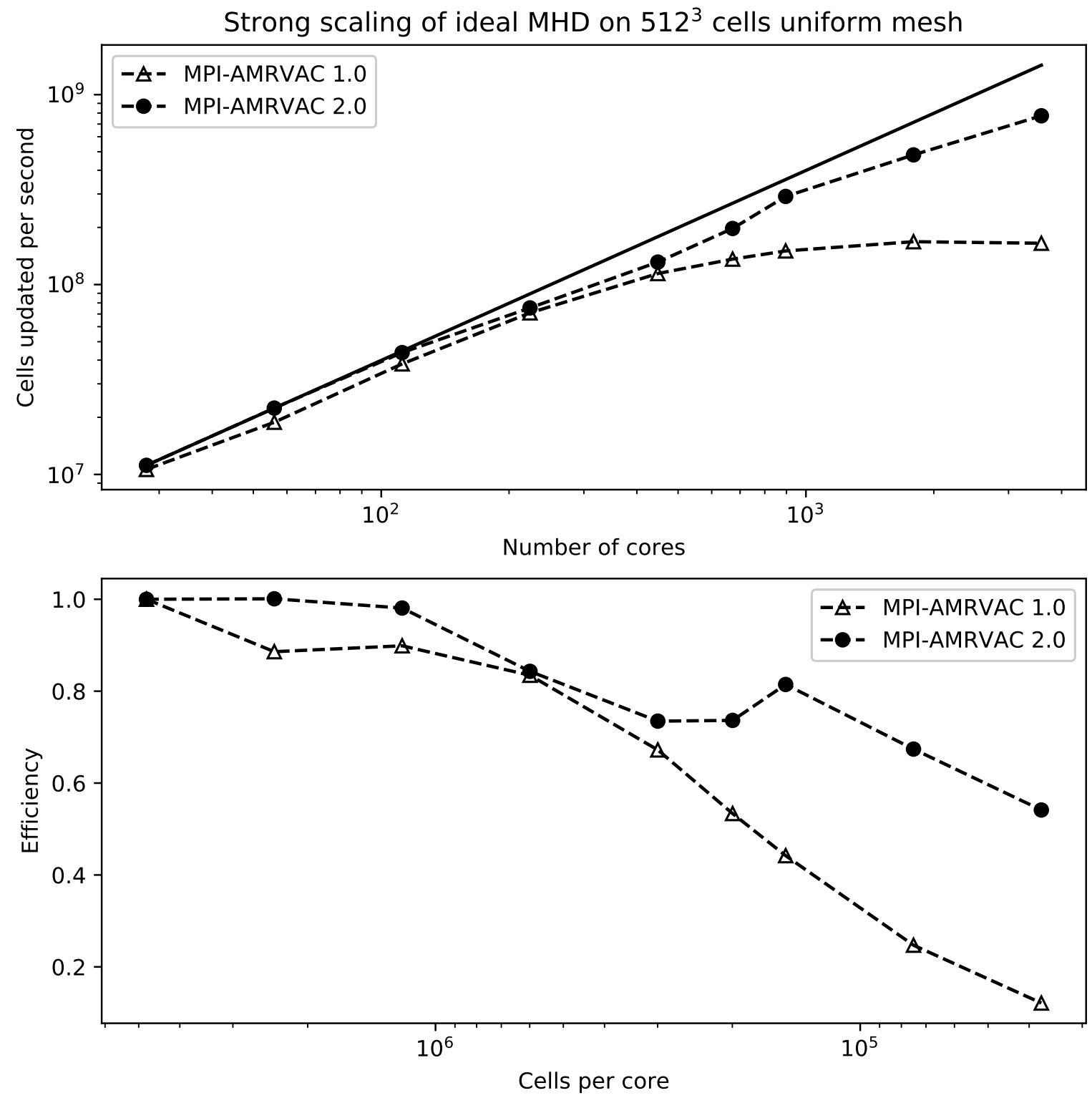

Figure 17. Strong scaling of an ideal MHD application with $512^{3}$ cells uniform mesh for MPI-AMRVAC 2.0 shown in dots and for MPI-AMRVAC 1.0 shown in empty trangles. (a) Cells updated per second for increasing number of cores. The solid line shows the ideal scaling case. (b) Efficiency of the speed up, shown as function of the number of cells per core.

- perform vectorization (e.g., loop over components),

- define parameters for specific applications,

- turn parts of the code on or off.

This extended use of the preprocessor made it harder to understand the code, especially as more and more features were added. The preprocessor in MPI-AMRVAC 2.0 has therefore been simplified; the only flags it now supports relate to dimensionality of the problem. Hence, the preprocessor is now only used to translate dimension-independent code 

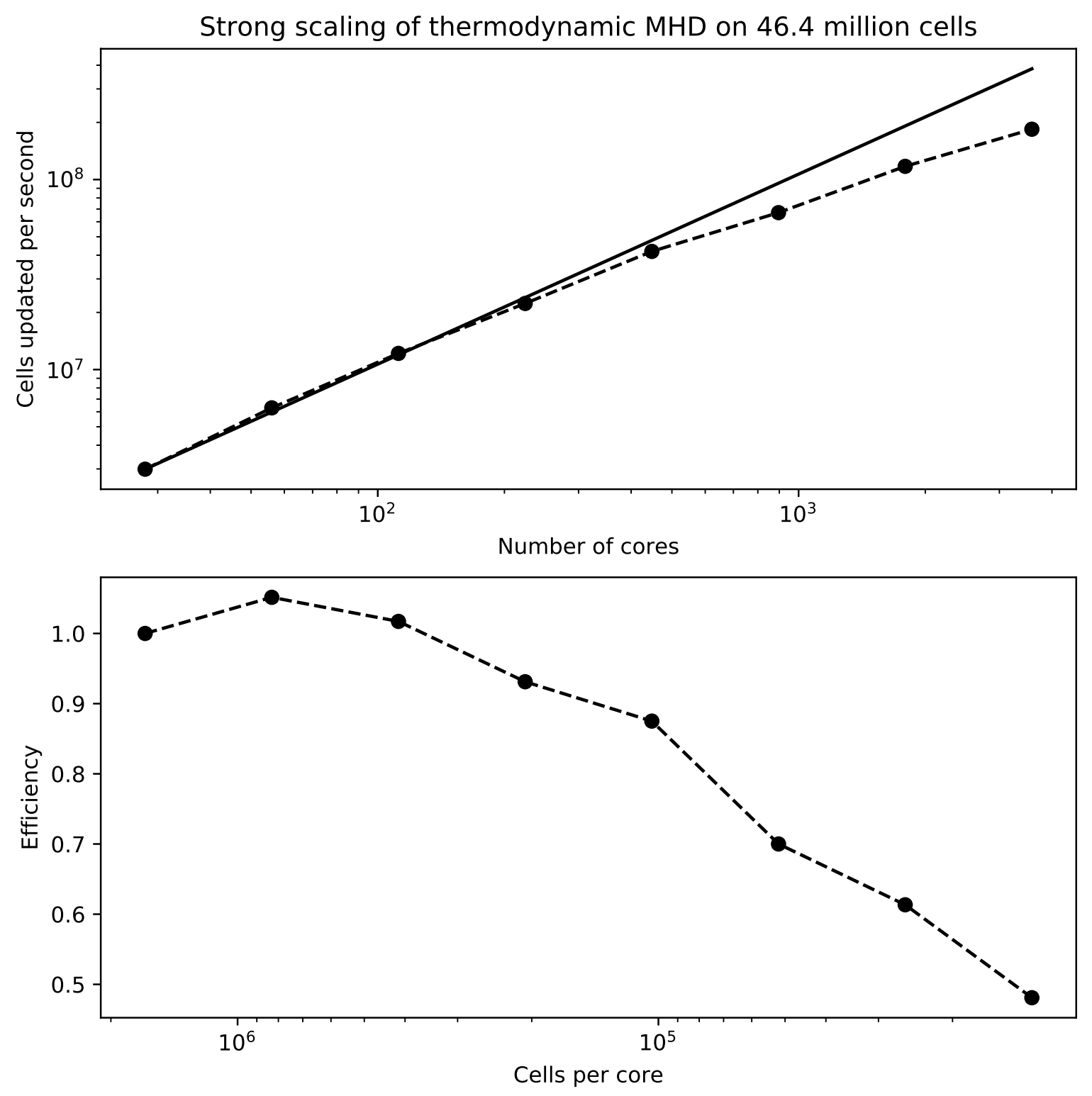

Figure 18. Strong scaling of a thermodynamic AMR-MHD application with 46.4 million cells. (a) Cells updated per second for increasing number of cores in dots connected with a dashed line. The solid line shows the ideal scaling. (b) Efficiency of the speed up, shown as function of the number of cells per core.

to $1 \mathrm{D}, 2 \mathrm{D}$ or 3D. This simplification of the preprocessor also required another modernization: instead of source files that were optionally included as flags were turned on or off, we now make use of standard Fortran modules. This made it possible to compile the full framework into a static library, including all the physics modules at once. This helps to ensure code stays compatible (and compiles) whenever changes are made, and significantly reduces compilation times. Furthermore, parallel compilation is now supported.

The modifications imply that the user program now has to specify which type of major physics module to activate, for example the hydrodynamics or magnetohydrodynamics one (previously handled through the preprocessor and before compilation). As all modules are now available in the library version, this raises awareness of users to the 
full framework functionality, and each physics module now comes with its own settings and parameters, for example whether or not an energy equation should be used, or how many tracers (or dust species in coupled gas-dust hydro problems) to be included. Thanks to that, users can switch on/off special functionalities, like thermal conduction, radial stretching grid, particles etc., by just choosing appropriate parameters in an input parameter file without wasting time on recompilation, which was needed more frequently in the old code. Furthermore, we provide extensive support for customization to a large variety of applications by using function pointers. A user has to provide a routine for setting initial conditions, but can also specify custom routines for boundary conditions, additional source terms, custom output routines etc. There have also been many smaller changes to facilitate users, most importantly are those related to handling the coordinate system explicitly (by selecting e.g. Cartesian 2D or 2.5D, or cylindrical, polar up to spherical 3D). Methods are encoded to automatically use the right number of ghost cells and the MPI-AMRVAC 2.0 settings to run an actual application can now be specified in multiple parameter files.

\subsection{Automatic regression tests}

A very important addition concerns regression tests for MPI-AMRVAC, in which their output is compared to stored 'correct' results. We intend to have a least one such test for each type of major functionality that the code supports, varying dimensionality, equations to solve, or choice of algorithmic approach. The primary goal of these tests is to ensure that code changes do not affect the result of existing simulations. They can also help new users to explore MPI-AMRVAC's functionality, and to detect bugs and incompatibilities.

The implementation of these tests is as follows. First, a standard simulation is scaled down so that it can be run in a short time, e.g. one to ten seconds on a standard machine. We then specify the program to produce a log file of type 'regression_test', which contains $\int w_{i} d V$ and $\int\left|w_{i}\right|^{2} d V$ over time, where $\vec{w}$ is a vector of the $n_{w}$ (typically conservative) physical variables $w_{i}$ per cell, for all variables $i \in\left[1, n_{w}\right]$. By comparing both the volume-integrated values of both cell-values and their squares, errors in conservation properties and almost all errors in the shape of the solution are detected. The resulting log file is then compared to a previously stored version, using a finite error threshold in comparing values $a$ to $b$ as in:

$$
|a-b| \leq \epsilon_{\mathrm{abs}}+\epsilon_{\mathrm{rel}}(|a|+|b|) / 2,
$$

where we by default use an absolute tolerance of $\epsilon_{\mathrm{abs}}=10^{-5}$ and relative tolerance of $\epsilon_{\mathrm{rel}}=10^{-8}$. These tolerances account for changes due to numerical roundoff errors. Roundoff errors can for example occur when computations are re-ordered (using different compilation flags and/or compilers) or when a different number of processors is used. Most of the tests we have are performed for several combinations of numerical schemes. They can be executed automatically by running 'make' in the tests folder of MPI-AMRVAC.

\subsection{Doxygen documentation}

Having good documentation can increase the efficiency of both users and maintainers. However, as it takes considerable effort to write such documentation, this remains an ongoing process. For new contributions, we follow these guidelines for inline documentation:

- Provide a short description of a non-trivial function/subroutine,

- When a piece of code is hard to understand, briefly describe what it does,

- Variable names should be self-explanatory without getting lengthy.

For MPI-AMRVAC 2.0 we make use of Doxygen (see http://www.doxygen.org), so that most documentation can be written close to the code it documents. This helps when reading the code, but also makes it simpler to update documentation when changing code. The documentation is automatically generated every day and published on http://amrvac.org, which provides a starting point for new users.

\subsection{New binary format}

Binary files are used to restart simulations, and they can also be converted to formats supported by visualization toolkits. For MPI-AMRVAC 2.0, we revised the binary format to make it future proof and simpler to use. The new format is described in Table 2. Below we briefly list some of the changes:

- The introduction of a version number to provide backwards functionality. 
MPI-AMRVAC 2.0

\begin{tabular}{|c|c|c|}
\hline description & variable name & data type \\
\hline \multicolumn{3}{|c|}{ Header } \\
\hline $\begin{array}{c}\text { version number } \\
\text { offset tree info } \\
\text { offset block data } \\
\text { number of variables } \\
\text { dimension of vectors } \\
\text { dimension of grid } \\
\text { maximum refinement level } \\
\text { number of grid leaves } \\
\text { number of grid parents } \\
\text { time iteration count } \\
\text { simulation time } \\
\text { min. coordinates } \\
\text { max. coordinates } \\
\text { resolution of base-level mesh } \\
\text { resolution of a block } \\
\text { geometry name } \\
\text { names of variables } \\
\text { name of physics } \\
\text { number of physics parameters } \\
\text { parameters } \\
\text { parameter names }\end{array}$ & $\begin{array}{c}\text { domain_nx } \\
\text { block_nx } \\
\text { nparams }\end{array}$ & $\begin{array}{c}\text { int32 } \\
\text { int32 } \\
\text { int32 } \\
\text { int32 } \\
\text { int32 } \\
\text { int32 } \\
\text { int32 } \\
\text { int32 } \\
\text { int32 } \\
\text { int32 } \\
\text { float64 } \\
\text { float64(ndim) } \\
\text { float64(ndim) } \\
\text { int32(ndim) } \\
\text { int32(ndim) } \\
\text { character(16) } \\
\text { character(nw*16) } \\
\text { character(16) } \\
\text { int32 } \\
\text { float64(nparams) } \\
\text { character(nparams*16) }\end{array}$ \\
\hline \multicolumn{3}{|c|}{ Tree information } \\
\hline $\begin{array}{l}\text { leaf or parent } \\
\text { refinement level } \\
\text { spatial index } \\
\text { block offset } \\
\end{array}$ & & $\begin{array}{c}\text { logical(nleafs+nparents) } \\
\text { int32(nleafs }) \\
\text { int32(ndim * nleafs) } \\
\text { int64(nleafs }) \\
\end{array}$ \\
\hline \multicolumn{3}{|c|}{ Block 1} \\
\hline $\begin{array}{l}\text { number of lower ghost cells } \\
\text { number of upper ghost cells } \\
\text { block data }\end{array}$ & $\begin{array}{l}\text { glo } \\
\text { ghi }\end{array}$ & $\begin{array}{c}\text { int32(ndim) } \\
\text { int32(ndim) } \\
\text { float64(1-glo:block_nx+ghi, nw })\end{array}$ \\
\hline & Block 2 & \\
\hline
\end{tabular}

Table 2. Description of the file format for binary files, in order of appearance in the file.

- The header is now at the start of the file, to simplify reading files.

- Byte offsets pointing to the location of the tree information and the block data allow new variables to be added at the end of the header.

- For each block, we also store its refinement, spatial index in the grid and the location of its data, to facilitate reading in data.

- Each grid block can now also store ghost cells.

\section{CONCLUSIONS AND OUTLOOK}

We provide an update on the open source software MPI-AMRVAC (Porth et al. 2014c), suited for parallel, grid adaptive computations of hydrodynamic and MHD astrophysical applications. Motivated by recent applications in the context of transonic accretion flows onto compact objects, or trans-Alfvénic solar wind outflows, we combine grid stretching principles with block-adaptivity. Its advantage is demonstrated in $1 \mathrm{D}, 2 \mathrm{D}$ and $3 \mathrm{D}$ scenarios, on spherically symmetric Bondi accretion, steady planar Bondi-Hoyle-Lyttleton flows, 3D wind accretion in Supergiant X-ray binaries, and 2D solar wind. In especially solar physics oriented MHD applications, we show the benefits of a generalization of the original potential magnetic field splitting approach, which allows splitting off any magnetic field. This is demonstrated in tests with a 3D static force-free magnetic flux rope, in settings with a 2D magnetic null-point, and in resistive MHD 
scenarios about magnetic reconnection in current sheets with either uniform or anomalous resistivity. The latter revisits a pioneering solar flare application with inclusion of anisotropic thermal conduction by Yokoyama \& Shibata (2001), which can now be simulated at more realistic low plasma beta conditions. We provide details on our implementation for treating anisotropic thermal conduction in multi-dimensional MHD applications, generalizing slope limited symmetric schemes by Sharma \& Hammett (2007) from 2D to 3D configuration, and ring diffusion tests that demonstrate its good accuracy and robustness in preventing from unphysical thermal flux from which all traditional schemes can not avoid. The new version of the code is shown to scale much better than the old one and its strong scaling is satisfactory up to 2000 cores in AMR simulations on 3D solar coronal rain driven by thermal instabilities. Generic improvements are also reported: modularization and monolithic compilation into a static library, the handling of automatic regression tests, the use of inline/online Doxygen documentation, and a new future-proof data format for input/output and restarts of simulations.

The code is freely available on http://amrvac.org, and can be used for a fair variety of hydro to magnetohydrodynamic computations. In a forthcoming paper (Ripperda et al., in prep.), we will document the code capability to solve for particle dynamics using a variety of solvers for handling the governing Lorentz equation, or by using a set of ordinary differential equations for quantifying their gyro-averaged motion. This can be done in static, as well as in dynamic electro-magnetic fields obtained from MHD applications, as recently demonstrated in Ripperda et al. (2017a,b). In combination with the capabilities documented in this paper, these improvements prepare for future applications of violent solar eruptive phenomena, and reconnection physics and particle acceleration associated with them. In the longer term, we may envision handling Poisson problems on the AMR hierarchy, or improving the treatment of radiative losses beyond the simplistic optically thin assumption.

C.X., J.T., I.E.M., and E.C. want to thank FWO (Research Foundation Flanders) for the award of postdoctoral fellowship to them. This research was supported by FWO and by KU Leuven Project No. GOA/2015-014 and by the Interuniversity Attraction Poles Programme by the Belgian Science Policy Office (IAP P7/08 CHARM). The simulations were conducted on the VSC (Flemish Supercomputer Center funded by Hercules foundation and Flemish government).

\section{REFERENCES}

Bisnovatyi-Kogan, G. S., Kazhdan, Y. M., Klypin, A. A., Lutskii, A. E., \& Shakura, N. I. 1979, Soviet Astronomy, 23

Bondi, H. 1952, MNRAS, 112

Bondi, H. \& Hoyle, F. 1944, MNRAS, 104, 273

Chané, E., Jacobs, C., Van der Holst, B., Poedts, S., \& Kimpe, D. 2005, A\&A, 432, 331

Chané, E., Poedts, S., \& Van der Holst, B. 2008, A\&A, 492, L29

Chen, Z., Frank, A., Blackman, E. G., Nordhaus, J., \&

Carroll-Nellenback, J. 2017, MNRAS, 468, 4465

Cowie, L. L. \& McKee, C. F. 1977, ApJ, 211, 135

Cunningham, A. J., Frank, A., Varnière, P., Mitran, S., \& Jones, T. W. 2009, ApJS, 182, 519

Edgar, R. G. 2004, New Astronomy Reviews, 48, 843

El Mellah, I. 2016, PhD thesis

El Mellah, I. \& Casse, F. 2015, MNRAS, 454, 2657

Fang, X., Xia, C., \& Keppens, R. 2013, ApJL, 771, L29

Fang, X., Xia, C., Keppens, R., \& Van Doorsselaere, T. 2015, ApJ, 807, 142

Feng, X., Zhang, S., Xiang, C., Yang, L., Jiang, C., \& Wu, S. T. 2011, ApJ, 734, 50

Foglizzo, T. \& Ruffert, M. 1996, A\&A, 361, 22

Fromang, S., Hennebelle, P., \& Teyssier, R. 2006, A\&A, 457, 371

Gombosi, T. I., Tóth, G., De Zeeuw, D. L., Hansen, K. C., Kabin, K., \& Powell, K. G. 2002, Journal of Computational Physics, 177,176

Groth, C., De Zeeuw, D. L., Gombosi, T. I., \& Powell, K. G. 2000, Journal of Geophysical Research: Space Physics, 105, 25053

Günter, S., Yu, Q., Krüger, J., \& Lackner, K. 2005, Journal of Computational Physics, 209, 354

Guo, X. 2015, Journal of Computational Physics, 290, 352
Guo, X., Florinski, V., \& Wang, C. 2016a, Journal of Computational Physics, 327, 543

Guo, Y., Xia, C., \& Keppens, R. 2016b, ApJ, 828, 83

Guo, Y., Xia, C., Keppens, R., \& Valori, G. 2016c, ApJ, 828, 82

Harten, A., Lax, P. D., \& van Leer, B. 1983, SIAM Review, 25, 35

Hendrix, T. \& Keppens, R. 2014, A\&A, 562, A114

Hendrix, T., Keppens, R., van Marle, A. J., Camps, P., Baes, M., \& Meliani, Z. 2016, MNRAS, 460, 3975

Hoyle, F. \& Lyttleton, R. A. 1939, Mathematical Proceedings of the Cambridge Philosophical Society, 35, 405

Jacobs, C., Poedts, S., Van der Holst, B., \& Chané, E. 2005, A\&A, 430, 1099

Keppens, R., Meliani, Z., van der Holst, B., \& Casse, F. 2008, A\&A, 486, 663

Keppens, R., Meliani, Z., van Marle, A. J., Delmont, P., Vlasis, A., \& van der Holst, B. 2012, Journal of Computational Physics, 231, 718

Keppens, R., Nool, M., Tóth, G., \& Goedbloed, J. P. 2003, Computer Physics Communications, 153, 317

Keppens, R. \& Porth, O. 2014, Journal of Computational and Applied Mathematics, 266, 87

Leroy, M. H. J. \& Keppens, R. 2017, Physics of Plasmas, 24, 012906

Lohner, R. 1987, Computer Methods in Applied Mechanics and Engineering, 61, 323

Low, B. C. 1977, ApJ, 212, 234

MacNeice, P., Olson, K. M., Mobarry, C., de Fainchtein, R., \& Packer, C. 2000, Computer Physics Communications, 126, 330

Manchester, W. B., Gombosi, T. I., Roussev, I., Ridley, A., de Zeeuw, D. L., Sokolov, I. V., Powell, K. G., \& Tóth, G. 2004, Journal of Geophysical Research (Space Physics), 109, A02107 
Mei, Z. X., Keppens, R., Roussev, I. I., \& Lin, J. 2017, A\&A, 604 L7

Meliani, Z. \& Keppens, R. 2010, A\&A, 520, L3

Meliani, Z., Keppens, R., Casse, F., \& Giannios, D. 2007, MNRAS, 376, 1189

Meyer, C. D., Balsara, D. S., \& Aslam, T. D. 2012, MNRAS, 422, 2102

Mignone, A., Zanni, C., Tzeferacos, P., van Straalen, B., Colella, P., \& Bodo, G. 2012, ApJS, 198, 7

Monceau-Baroux, R., Porth, O., Meliani, Z., \& Keppens, R. 2014, A\&A, 561, A30

Nool, M. \& Keppens, R. 2002, Comp. Meth. Appl. Math., 2, 92

Parrish, I. J. \& Stone, J. M. 2005, ApJ, 633, 334

Phillips, J., Bame, S., Barnes, A., Barraclough, B., Feldman, W., Goldstein, B., Gosling, J., Hoogeveen, G., McComas, D., Neugebauer, M., et al. 1995, Geophysical Research Letters, 22, 3301

Porth, O., Komissarov, S. S., \& Keppens, R. 2014a, MNRAS, 443,547

-. 2014b, MNRAS, 438, 278

Porth, O., Olivares, H., Mizuno, Y., Younsi, Z., Rezzolla, L., Moscibrodzka, M., Falcke, H., \& Kramer, M. 2017,

Computational Astrophysics and Cosmology, 4, 1

Porth, O., Xia, C., Hendrix, T., Moschou, S. P., \& Keppens, R. 2014c, ApJS, 214, 4

Powell, K. G., Roe, P. L., Linde, T. J., Gombosi, T. I., \& De Zeeuw, D. L. 1999, Journal of Computational Physics, 154, 284

Powell, K. G., Roe, P. L., Linde, T. J., Gombosi, T. I., \& De Zeeuw, D. L. 1999, Journal of Computational Physics, 154, 284

Ripperda, B., Porth, O., Xia, C., \& Keppens, R. 2017a, MNRAS, 467,3279

-. 2017b, MNRAS, 471, 3465

Rossmanith, J. A. 2004, Computer Physics Communications, 164, 128
Ruffert, M. 1994, ApJ, 427, 342

Sharma, P. \& Hammett, G. W. 2007, Journal of Computational Physics, 227, 123

Stone, J. M., Gardiner, T. A., Teuben, P., Hawley, J. F., \& Simon, J. B. 2008, ApJS, 178, 137

Tanaka, T. 1994, Journal of Computational Physics, 111, 381

Toro, E. F., Spruce, M., \& Speares, W. 1994, Shock Waves, 4, 25

Tóth, G. 1997, Journal of Computational Physics, 138, 981

Tóth, G., Ma, Y., \& Gombosi, T. I. 2008, Journal of Computational Physics, 227, 6967

Tzeferacos, P., Fatenejad, M., Flocke, N., Graziani, C., Gregori, G., Lamb, D. Q., Lee, D., Meinecke, J., Scopatz, A., \& Weide, K. 2015, High Energy Density Physics, 17, 24

Čada, M. \& Torrilhon, M. 2009, Journal of Computational Physics, 228, 4118

van der Holst, B. \& Keppens, R. 2007, Journal of Computational Physics, 226, 925

van der Holst, B., Keppens, R., \& Meliani, Z. 2008, Computer Physics Communications, 179, 617

van Leer, B. 1974, Journal of Computational Physics, 14, 361

Vlasis, A., van Eerten, H. J., Meliani, Z., \& Keppens, R. 2011, MNRAS, 415, 279

Vreugdenhil, C. \& Koren, B. 1993, Numerical methods for advection-diffusion problems (Braunschweig: Vieweg), 117-138 Wang, P. \& Abel, T. 2009, ApJ, 696, 96

Xia, C., Chen, P. F., \& Keppens, R. 2012, ApJL, 748, L26

Xia, C. \& Keppens, R. 2016, ApJ, 823, 22

Xia, C., Keppens, R., Antolin, P., \& Porth, O. 2014, ApJL, 792, L38

Xia, C., Keppens, R., \& Fang, X. 2017, A\&A, 603, A42

Yokoyama, T. \& Shibata, K. 2001, ApJ, 549, 1160

Zhao, X., Xia, C., Keppens, R., \& Gan, W. 2017, ApJ, 841, 106

Ziegler, U. 2005, A\&A, 435, 385 